\title{
Heterogeneous-PAKE: Bridging the Gap between PAKE Protocols and Their Real-World Deployment
}

\author{
Rong Wei ${ }^{*}$ \\ State Key Laboratory of Information \\ Security, Institute of Information \\ Engineering, Chinese Academy of \\ Sciences \\ weirong1130@iie.ac.cn \\ Jiankuo Dong \\ School of Computer Science, \\ Nanjing University of Posts and \\ Telecommunications \\ djiankuo@njupt.edu.cn
}

\author{
Fangyu Zheng ${ }^{\dagger}$ \\ State Key Laboratory of Information \\ Security, Institute of Information \\ Engineering, Chinese Academy of \\ Sciences \\ zhengfangyu@iie.ac.cn
}

\section{Guang Fan*}

State Key Laboratory of Information

Security, Institute of Information

Engineering, Chinese Academy of

Sciences

fanguang@iie.ac.cn

\author{
Lili Gao* \\ State Key Laboratory of Information \\ Security, Institute of Information \\ Engineering, Chinese Academy of \\ Sciences \\ gaolili1994@iie.ac.cn
}

\section{Lipeng $\mathrm{Wan}^{*}$}

State Key Laboratory of Information Security, Institute of Information Engineering, Chinese Academy of Sciences wanlipeng@iie.ac.cn

\begin{abstract}
Jingqiang Lin
School of Cyber Security, University of Science and Technology of China

linjq@ustc.edu.cn
\end{abstract}

\begin{abstract}
Two entities, who only share a password and communicate over an insecure channel, authenticate each other and agree on a large session key for protecting their subsequent communication. This is called the password-authenticated key exchange (PAKE) protocol. PAKE protocol has been considered a suitable substitute for the prevailing hash-based authentication which is vulnerable to various attacks. However, vendors are discouraged by both its prohibitively computational overheads as well as integrating costs, leading to its limited use since being proposed.

After carefully analyzing the general workflow of PAKE protocols, we present Heterogeneous-PAKE, an entire PAKE stack with high-performance and compatibility for both client-side and server-side for Web systems. Using SRP and SPAKE2+ as case studies, we conduct a series of comprehensive experiments, especially comparing with the conventional hash-based solutions to evaluate the Heterogeneous-PAKE. The implementation harvests high throughput on the server-side with over $240 \mathrm{k}, 70 \mathrm{k}$, $30 \mathrm{k}$, and 1,650k operations per second for SRP-1024, SRP-1536, SRP-2048, and SPAKE2+ respectively. Meanwhile, on most testing platforms, the latency is well controlled within user-acceptable bounds, especially the SPAKE2+ whose delay is less than 3x that of a traditional authentication approach based on Bcrypt. The
\end{abstract}

* The authors are also with School of Cyber Security, University of Chinese Academy of Sciences, Beijing, China

${ }^{\dagger}$ Fangyu Zheng is the corresponding author.

Permission to make digital or hard copies of all or part of this work for personal or classroom use is granted without fee provided that copies are not made or distributed for profit or commercial advantage and that copies bear this notice and the full citation on the first page. Copyrights for components of this work owned by others than ACM must be honored. Abstracting with credit is permitted. To copy otherwise, or republish, to post on servers or to redistribute to lists, requires prior specific permission and/or a fee. Request permissions from permissions@acm.org. ACSAC '21, December 6-10, 2021, Virtual Event, USA

(c) 2021 Association for Computing Machinery.

ACM ISBN 978-1-4503-8579-4/21/12 ..\$15.00

https://doi.org/10.1145/3485832.3485877
Yuewu Wang*

State Key Laboratory of Information

Security, Institute of Information

Engineering, Chinese Academy of

Sciences

wangyuewu@iie.ac.cn

empirical results demonstrate that the Heterogeneous-PAKE is a very economical (with only a GPU-ready server) and convenient (with an easy-to-integrate software stack without user participation or database rebuilding) solution for upgrading existing systems with high-performance PAKE services.

\section{CCS CONCEPTS}

- General and reference $\rightarrow$ Performance; • Applied computing $\rightarrow$ Service-oriented architectures.

\section{KEYWORDS}

PAKE protocol, password authenticating, heterogeneous computing model, SRP, SPAKE2+

\section{ACM Reference Format:}

Rong Wei, Fangyu Zheng, Lili Gao, Jiankuo Dong, Guang Fan, Lipeng Wan, Jingqiang Lin, and Yuewu Wang. 2021. Heterogeneous-PAKE: Bridging the Gap between PAKE Protocols and Their Real-World Deployment. In Annual Computer Security Applications Conference (ACSAC '21), December 6-10, 2021, Virtual Event, USA. ACM, New York, NY, USA, 15 pages. https://doi.org/10.1145/3485832.3485877

\section{INTRODUCTION}

Passwords constitute the most ubiquitous means of authentication on the Internet, from the common to the most sensitive applications. A general password-based authentication practice relies on SSL/TLS [37] and the slow hash functions (e.g., Bcrypt [35] and Scrypt [33]): the user sends her (his) password to the server under over an SSL/TLS-protected channel, and then the server decrypts the password and verifies against a one-way image typically computed via slow hash functions. The password image of each user is stored on the server. There are two obvious disadvantages of this approach:

- Although storing the password images on the server-side, it is still vulnerable to dictionary attacks once the database leaks. [43] summarized 56 biggest data breaches, in 
which Twitter, Facebook and Yahoo, as famous international IT vendors in the world, were reported to be victims of data breaches, leading to compromises of 300 million to 3 billion users' private data, including plaintext passwords or hashed passwords. Worse still, since password reuse is rampant, any data breach would make those innocent, heavily-guarded sites potentially vulnerable. With credential stuffing techniques, attackers could easily expand damages to websites not compromised.

- The password (or its hashed image) in travel is exposed to eavesdroppers and man-in-the-middle (MitM) attacks [5]. Although most websites are protected by SSL/TLS connections, security breaks if the SSL/TLS channel is established with a compromised server's private key (a widespread concern given today's too-common PKI failures [39]). Even if the TLS channel was not corrupted, passwords would still be decrypted after passing through the TLS offload gateway, and then travel in plaintext through the internal network.

\subsection{PAKEs and the Challenges When Applied}

Considering these problems, researchers proposed password authenticated key exchange (PAKE) protocols [4], which are geared to prevent the above-mentioned attacks. As the name suggests, a PAKE protocol is intended to establish a strong session key for participants based on a (weak) password.

According to the knowledge of the password hold by each participant, a PAKE protocol can be labeled as symmetric (or balanced) PAKE (bPAKE) or asymmetric (or augmented) PAKE (aPAKE). While the password is shared by all of the participants, bPAKE protocols do not apply to the client-server setting. In contrast, an aPAKE protocol not only prevents passwords from eavesdropping on channels but from data leakages on the serverside. In this paper, we concentrate on aPAKE schemes, which are more suitable for Web applications. Compared with traditional password-based login, instead of authenticating the user by validating her (his) password with the hash stored on the serverside, an aPAKE protocol is characterized with the following two properties:

(1) Messages are randomized and thus, immune to insecure communication channels like the Internet.

(2) The server side does not keep a password or its hash, instead, it holds a much more complicated mapping (in other words, a verifier) of the password. The security strength of a verifier is based on mathematical hard problems, thus the passwords are protected against dictionary attacks [29].

Over the past three decades, PAKEs have been heavily studied but only been used in quite limited ranges compared with the huge number of websites. As far as we know, Apple employed SRP [47] for protecting iCloud security codes and recently used SPAKE2+ [42] for car keys security [15]. 1Password used SRP along with TLS/SSL to secure authentication between client and server without storing plaintext user passwords [12]. Meanwhile, other websites are barely reported for PAKE usage, Facebook [1] and Google [2] are even reported to store passwords in plaintext. The reasons for PAKE's limited real-world usage are manifold, among which performance issues and migration cost for the existent systems are the main concerns. Technical challenges include:
- Constructed over public-key cryptography, a PAKE protocol is characterized by far higher workloads than hashbased authentications.

- Besides significant performance penalties, vendors have to pay heavy taxes to upgrade their systems for PAKE service. In contrast to simple cryptographic primitives, e.g., digital signature and data encryption, a PAKE protocol consists of multiple roundtrips requiring costly modifications to the server-side.

- Compared with hash-based manners, an aPAKE protocol requires rich clients to perform complex computations, whereas diverse client-side environments bring more compatibility issues.

\subsection{Contributions}

In this paper, after carefully analyzing the general workflow of aPAKE protocols, we present Heterogeneous-PAKE, an entire PAKE stack for Web systems that offers high-performance and compatibility for both client-side and server-side, including cryptographic primitives, protocol implementations and network scheduling.

The main goal of this work is to bridge the gap between PAKE protocols and their real-world deployment at the lowest possible cost. More specifically, our contributions are four-fold:

(1) Firstly, we put forward an easy-to-integrate PAKE service framework for Web applications, minimizing the vendors' costs for system upgrading.

(2) Secondly, on the server side, we provide a full PAKE implementation in SIMD and SIMT computing fashions to process highly concurrent user requests. The server-side also integrates heterogeneous computing to the Web systems with minimal modifications by a finely scheduled network processing architecture. While the framework harvests low latency as well as high throughput, the extraordinary performance mainly comes from COTS products, including a desktop-class CPU and an NVIDIA graphics card.

(3) Thirdly, on the client side, we implement a JavaScriptbased library including the fundamental cryptographic primitives for both DL and EC-based PAKE, as well as the high-level PAKE protocols.

(4) Finally, targeting two representative aPAKE protocols, SRP and SPAKE2+, we build a high-performance aPAKE server and corresponding components for Web applications. The implementation harvests high throughput on the serverside with over $240 \mathrm{k}, 70 \mathrm{k}, 30 \mathrm{k}$ and $1,650 \mathrm{k}$ operations per second for SRP-1024, SRP-1536, SRP-2048 and SPAKE2+ respectively. In the meanwhile, the client-side's latency is well controlled within user-acceptable bounds, especially the SPAKE2+ implementation whose delay is less than $3 \mathrm{x}$ that of a traditional authentication approach based on Bcrypt and does not exceed $0.4 \mathrm{~s}$ on most platforms even facing the concurrency of over $1600 \mathrm{k}$ requests per second.

\subsection{Outline}

The next section begins with the background and requisite knowledge. Section 3 illustrates the overall framework of the prototype system. Section 4 details the design and optimizations we adopted on different aspects. In Section 5 we conduct a series of experiments and performance evaluations. Finally, we draw conclusions in Section 6. 


\section{BACKGROUND}

This section introduces basic knowledge of PAKE protocols, demonstrates two target protocols, and illustrates features of involved parallel platforms.

\subsection{PAKE Protocol}

A PAKE scheme is an interactive protocol that allows its participants to authenticate each other and agree on a shared cryptographic key with a (weak) password.

Augmented PAKE (aPAKE) protocols [17, 40, 42, 46] are further designed to keep an individual user's password secure against server compromise in client-server scenarios which are quite common in the real world.

Although PAKE protocol achieves strong security with weak passwords, it has been hampered from widespread use by performance issues. Besides expensive mathematical computations and multiple interactions, some schemes introduce additional complexity to circumvent existing patents. Table 1 lists the main overheads of representative aPAKE protocols, including the number of modular exponentiation for discrete logarithm (DL) setting or scalar multiplications for elliptic curve (EC) setting. Since the concrete operations of a PAKE scheme vary in different publications, our data comes from IEEE P1363.2 [13] and Internet drafts [42]. Particular attention is paid to fixed-base operations which tend to be accelerated with pre-computations. In order to solve this dilemma, we design an entire PAKE stack for Web systems and implement SRP-3 [47] and SPAKE2+ [42] as two case studies.

Table 1: Overhead of aPAKE Protocols

\begin{tabular}{c|c|cc|cc}
\hline \multirow{2}{*}{ Protocol } & \multirow{2}{*}{ Setting } & \multicolumn{2}{|c|}{ Client } & \multicolumn{2}{c}{ Server } \\
& & Fixed-base & Total & Fixed-base & Total \\
\hline AMP[21] & DL/EC & 2 & 4 & 0 & 3 \\
BSPEKE2[16] & DL/EC & 2 & 6 & 1 & 5 \\
PAKZ[23] & DL/EC & 1 & 2 & 1 & $2 / 1$ \\
SPAKE2+ & DL/EC & 1 & $8 / 6$ & 1 & $6 / 5$ \\
SRP-3 & DL & 2 & 3 & 1 & 3 \\
SRP-5[45] & EC & 2 & 4 & 1 & 3 \\
SRP-6[46] & DL & 2 & 3 & 1 & 3 \\
\hline
\end{tabular}

Secure Remote Password (SRP) protocol, a verifier-based PAKE scheme proposed by T Wu [47], is suitable for negotiating secure connections using a user-supplied password while eliminating the security problems traditionally associated with reusable passwords. Figure 1 presents steps of SRP-3 which take three round trips to finish a key exchange and effectively conveys a zeroknowledge password proof from the user to the server. As the most representative one of industrialized aPAKE protocols, SRP is used for account validation in keychain recovery and signing into shared devices in iPhone [15]. Besides, SRP is integrated into TLS [46].

SPAKE2+ is the augmented version of SPAKE2 [3] protocol which is compatible with any prime order group and is proven secure in the Universal Composable (UC) model [6]. While SRP works on a ring and is hence uneasy for transplanting to an EC group, SPAKE2+ is EC-compatible and does not require any full domain hash function or ideal ciphers onto EC groups. When implemented with EC, the protocol runs as Figure 2 shows. The verifier of SPAKE2+ consists of an element $w_{0}$ on $G F(p)$ and an

\begin{tabular}{|c|c|c|c|}
\hline & Client & & Server \\
\hline 1. & Input $(I D, \pi)$ & $\stackrel{I D}{\rightarrow}$ & $($ lookup $s, v)$ \\
\hline 2. & $x=H(s, \pi)$ & $\stackrel{s}{\leftarrow}$ & \\
\hline 3. & $A=g^{a}$ & $\stackrel{A}{\rightarrow}$ & \\
\hline 4. & & $\stackrel{B, u}{\leftarrow}$ & $B=v+g^{b}$ \\
\hline 5. & $S=\left(B-g^{x}\right)^{a+u x}$ & & $S=\left(A v^{u}\right)^{b}$ \\
\hline 6. & $K=H(S)$ & & $K=H(S)$ \\
\hline 7. & $M_{1}=H(A, B, K)$ & $\stackrel{M_{1}}{\longrightarrow}$ & (verify $M_{1}$ ) \\
\hline 8. & (verify $M_{2}$ ) & $\stackrel{M_{2}}{\leftarrow}$ & $M_{2}=H(A, B, K)$ \\
\hline \multicolumn{4}{|c|}{$\begin{array}{l}H: \text { hash function } s: \text { the user's salt stored on server side } \\
\pi: \text { the user's password } g: \text { a generator of } \operatorname{GF}(n) \\
x: \text { private key derived from } s \text { and } \pi\end{array}$} \\
\hline \multicolumn{4}{|c|}{$\begin{array}{l}I D \text { : the user's identity } v: v e r i f i e r \text { derived from } g \text { and } \\
x \text {, computed as } v=g^{x} \text {, stored on server side }\end{array}$} \\
\hline
\end{tabular}

Figure 1: the Workflow of SRP3

EC point $L$ computed as $L=\left[w_{1}\right] P$, where $w_{1}$ together with $w_{0}$ are derived from the password $\pi$, and $P$ is a generator of the EC group $G$.

\begin{tabular}{|c|c|c|c|}
\hline \multicolumn{3}{|c|}{ Client } & Server \\
\hline 1. & Input $(I D, \pi)$ & $\stackrel{I D}{\rightarrow}$ & \multirow{4}{*}{ (lookup $\left.w_{0}, L\right)$} \\
\hline 2. & $\left(w_{0 s}, w_{1 s}\right)=P B K D F(\pi)$ & \multirow[t]{3}{*}{$\stackrel{*}{\leftarrow}$} & \\
\hline 3. & $w_{0}=w_{0 s} \bmod p$ & & \\
\hline 4. & $w_{1}=w_{1 s} \bmod p$ & & \\
\hline 5. & $X=[x] P+\left[w_{0}\right] M$ & \multirow{4}{*}{$\underset{\leftarrow}{\stackrel{X}{\longrightarrow}}$} & $Y=[y] P+\left[w_{0}\right] N$ \\
\hline 6. & $Z=[h x]\left(Y-\left[w_{0}\right] N\right)$ & & $Z=[h y]\left(X-\left[w_{0}\right] M\right)$ \\
\hline 7. & $V=\left[h w_{1}\right]\left(Y-\left[w_{0}\right] N\right)$ & & $V=[h y] L$ \\
\hline 8. & $K_{a} \| K_{e}=H(T T)$ & & $K_{a} \| K_{e}=H(T T)$ \\
\hline 9. & $K_{c A} \| K_{c B}=K D F(K a)$ & \multirow{2}{*}{$\stackrel{K_{c A}}{\longrightarrow}$} & (verify $K_{c A}$ ) \\
\hline 10. & (verify $K_{c B}$ ) & & $K_{c A} \| K_{c B}=K D F(K a)$ \\
\hline \multirow{3}{*}{\multicolumn{4}{|c|}{$\begin{array}{l}\pi: \text { the user's password } \quad L: \text { the verifier of } \pi, \text { computed as } L=\left[w_{1}\right] P \\
H: \text { hash function } I D: \text { the user's identity, such as username and Email } \\
P: \text { a generator of } G \quad h: \text { the cofactor of } G\end{array}$}} \\
\hline & & & \\
\hline & & & \\
\hline \multicolumn{4}{|c|}{$\begin{array}{l}M \text { and } N \text { are fixed elements of } G \quad x \text { and } y \text { are one-time random elements. } \\
T T: \text { concatenation of } I D \text {, Server, } M, N, X, Y, Z, V, w_{0}\end{array}$} \\
\hline
\end{tabular}

Figure 2: the Workflow of SPAKE2+

\subsection{Parallel Platforms}

Facing up with the growing demand for greater computing powers across industry segments, vendors tend to equip their processors with vector instruction sets, such as AMD 3DNow [30], ARM NEON [36], etc. Intel also announced its MMX/SSE/AVX [18, 32] series. A vector instruction performs the same operation to multiple elements resided on a vector register in parallel, which is also known as single instruction, multiple data (SIMD). As the latest, also the most powerful version of the AVX series up to now, AVX-512 [7] provides 8-way 64-bit (or 16-way 32-bit) instructions which are of great importance for multi-precision arithmetic in the public-key cryptosystem.

While vector instruction brings several-fold speedup to CPU programs, this is far from dealing with highly concurrent tasks. Consequently, researchers attempt to exploit the powerful computing ability of graphic processing units (GPUs) to handle massive requests effectively. 
GPUs consist of a large number of cores, processing parallel tasks by broadcasting an instruction to every executing unit, which is called single instruction, multiple threads (SIMT). When used for computations other than graphics rendering, GPUs act as a co-processor with built-in memory and processing units. NVIDIA released Compute Unified Device Architecture (CUDA) [38], a programming framework that enables developers to dramatically speed up computing applications by harnessing the power of GPUs. Some excellent works for GPUaccelerated computation have been delivered in recent years [8$11,31]$.

\section{OVERVIEW}

As we aim to provide efficient aPAKE services for Web applications, this section illustrates our solution and considerations.

\subsection{Design Goal}

Since our goal is proposing a solution for integrating aPAKE services to existing Web applications (which may be a large-scale website with massive user data), instead of building a new website with aPAKE functionality, some trivial issues should be taken into account. The main requirements of our scheme include:

- easy-to-integrate. The vendors could upgrade their applications with minimal costs.

- extensible. It should be convenient to extend the system to clustered Web servers, and it should not produce an extra price when the cluster scale grows.

- high-performance. The system shall adapt to dynamic workloads. Concretely, it achieves high throughput when facing high concurrency requests and low latency for sporadic tasks.

\subsection{Workflow Analysis}

PAKE schemes are characterized by multiple interactions between the participants in a single execution. Despite the complexity, the standard authentication of an industrialized aPAKE protocol could be summarized as Figure 3, which consists of three general stages:

(1) preamble exchange. In this stage, the user types in her (or his) ID and password in a form. Then the ID and negotiable information $\left(\operatorname{In} f_{C}\right)$ like cipher suite and protocol version are sent to the server-side. Upon reception of the ID, the server retrieves its verifier (and salt if necessary) from the database accordingly and returns requisite information $\left(\operatorname{Inf} f_{S}\right)$ to the client-side.

(2) protocol setup. By exchanging key derivation materials $\left(K D_{C}\right.$ and $\left.K D_{S}\right)$, participants compute their shared secret and then derive session keys $\left(K_{C}\right.$ or $\left.K_{S}\right)$. The process contains at least one data exchange. $K_{C}$ and $K_{S}$ will be equal if the protocol succeeds. For the server-side, all mathematical computations occur in this stage.

(3) key confirmation (optional). Participants, especially servers, are required to confirm the clients' knowledge of the session key before putting it into use. The verification tends to be finished with a hash-based scheme.

A stage does not always mean a roundtrip or an interaction, since there may be multiple roundtrips at a single stage (especially the second phase) of a few protocols. Even for the same PAKE protocol, its communication overhead varies in different publications, e.g. academic papers, standard documents. In this

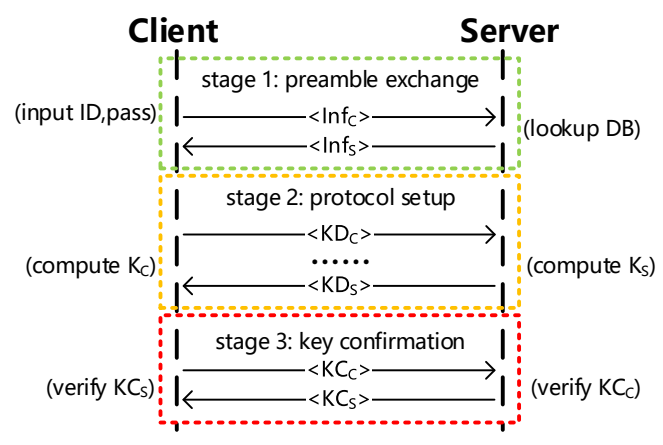

Figure 3: Protocol Flow of aPAKE, where $K$ means "Key", $K D$ means "Key Derivation", and $K C$ means "Key Confirmation"

work, we implement protocols according to their definitions in IEEE P1363.2 or Internet drafts.

When integrated into Web applications, there is a possibility for further simplifying an aPAKE protocol to two stages without a security breach. Since a Web browser downloads JavaScript programs which hard code co-parameters from the server over HTTP(S) on opening URL, parameter negotiation is no longer a prerequisite. The rest of the preamble exchange could be postponed to the next stage if $K D_{C}$ in stage 2 is independent to $\operatorname{Inf} f_{S}$ in stage 1.

Both the two protocols we select for implementation are of three rounds and satisfy the above assumption. Figure 4 shows the original workflow of a 3-round aPAKE protocol and the modified version we proposed for Web systems. The preamble exchange is replaced with the requisite preload of JavaScript and a manual HTTP request. Mathematical computations of server-side aPAKE centralize within the dotted border, which could be finished with a plug-in or an independent server.

A plug-in runs on the target device as a software add-on and has a great advantage of responding speed over remote services for its relatively short invocation path. However, a complicated plug-in brings a noticeable performance penalty with too much occupation of native resources, especially when facing massive tasks. When it comes to integrating a computational expensive service (e.g. SSL management) into existent systems, an independent server is preferred for lower upgrade costs and better performance in high concurrent situations. While aPAKE protocols are computationally intensive and thus naturally suitable for remote serving mode, we provide aPAKE service remotely with a stand-alone server (referred to as PAKE-Server later in this paper). Another consideration is that most COTS servers are headless devices that have no space for a powerful discrete graphics card required by our scheme.

More specifically, when a user opens the website, the browser downloads static resources including the JavaScript program with aPAKE functions. Then the user inputs her (or his) username $I D$ and password $P$ in a form and clicks the "Submit" button Subsequently, the authentication is carried out as follows:

(1) The Web browser computes $K D_{C}$ and necessary intermediate variables with $I D$ and $P$ and sends $K D_{C}$ together with $I D$ to the Web server.

(2) The Web server looks up the user's verifier $v$ (and salt $s$, if any) from the database. If the retrieval succeeds, it establishes a session for the user and sends $v$ and requisite data in $K D_{C}$ to PAKE-Server. 


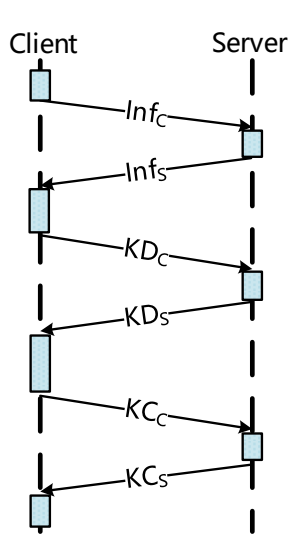

(a) Original Workflow

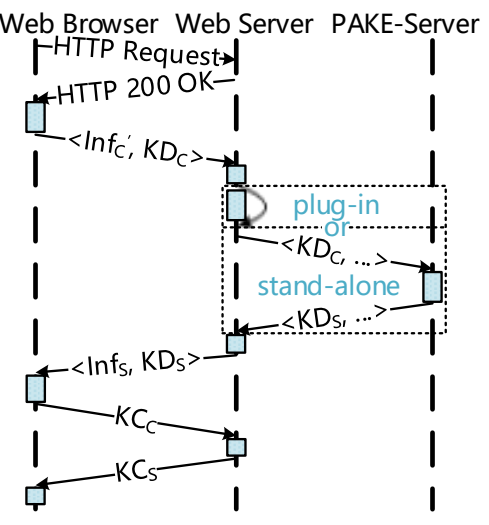

(b) Tweaked Workflow for Web System
Figure 4: Workflow of 3-round aPAKE Protocols

(3) PAKE-Server computes $K D_{S}$ as well as other requisite materials for key derivation and returns them to the Web server.

(4) The Web server derives session key $K_{S}$ based on data received, and then replies to the browser with $K D_{S}$.

(5) The Web browser derives session key $K_{C}$, then computes and sends key confirmation material $K C_{C}$ to the Web server.

(6) The Web server computes key confirmation material $K C_{S}$, verifies the equivalence of $K_{C}$ and $K_{S}$ with $K C_{C}$ and $K C_{S}$, and then sends $K C_{S}$ back to the browser. If the key confirmation fails, it destroys the session and clears all variables cached before.

(7) The Web browser confirms the session key based on $K C_{C}$ and $K C_{S}$. If $K_{C}=K_{S}$, then output $K_{C}$, otherwise the authentication fails.

\section{PAKE IMPLEMENTATION}

Taking SRP and SPAKE2+ as case studies, this section details the implementations of our scheme in a bottom-up manner. We firstly optimize fundamental primitives for CPU, GPUs as well as Web browsers. Then a heterogeneous protocol stack is constructed over the primitives. After that, we illustrate the up-level design of the PAKE-Server, including I/O processing and task dispatching. Finally, we present the overall framework and give guidelines for applying the scheme to existing Web systems.

\subsection{Cryptographic Primitive Optimizations}

We provide implementations of each protocol in JavaScript for Web browser, C (with AVX-512 instructions) and CUDA C for PAKE-Server respectively. The task is decomposed into three levels as shown in Figure 5 from a developer's perspective. More specifically, SRP is constructed over $G F(p)$ where $p$ tends to be a generalized prime, and thus Montgomery multiplication [27] is required for modular multiplication and exponentiation. By comparison, we implement SPAKE2+ over Edwards25519 [14] group, which could further be decomposed into $G F(p 25519)$ operations where $p 25519=2^{255}-19$. While $p 25519$ is a pseudo-Mersenne prime, the modular operation could be accelerated with fast reduction algorithm.

4.1.1 JavaScript Implementation. The client-side program is reconstructed over the open-source JavaScript library sjcl firstly

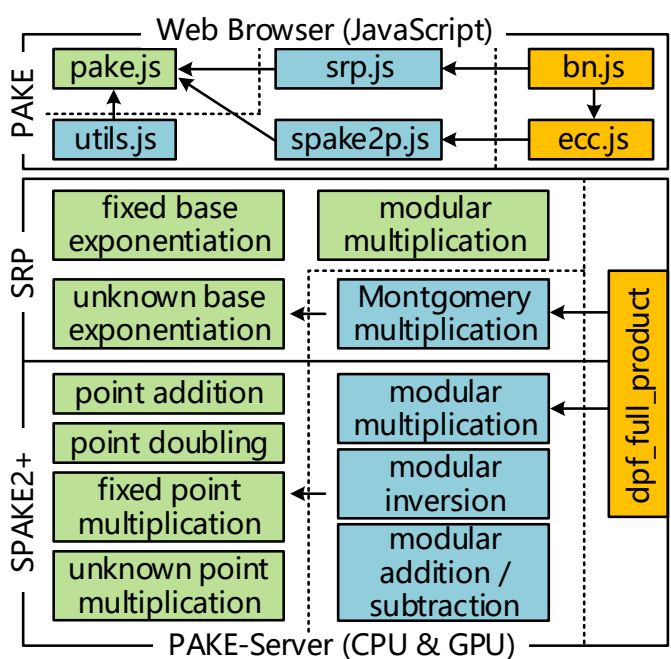

Primitive Level: high mid low

Figure 5: Key Primitives

released by Stark et. al. [41] in 2009. Considering the browser backward compatibility, the client-side protocols are performed by pure JavaScript-based implementation. Despite the excellent compatibility, JavaScript is naturally much slower than native languages and needs to download on each visit to the website. Therefore, the code size should be rigorously restricted to reduce transmission delay. Worse still, affected by the single-thread mode of JavaScript, any synchronized executing would block the page rendering, and hence, we do not suggest time-consuming pre-computations. While the Web Worker API [34] seems to be able to offer acceleration in a background thread, its structured clone brings non-negligible delay to cryptography computations.

Consequently, we decompose pake.js into several modules as shown in Figure 5. The basic level consists of two modules, $b n . j s$ implements finite field operations, and ecc.js provides a framework for ECC functionalities. Both the two are built-in modules of $s j c l . j s$, and $b n . j s$ is also a building block for ecc.js. While ecc.js only provides Weiertrass curves, we additionally implement Edwards25519 curve with extended coordinate system based on the framework.

The instant downloading mode prohibits offline pre-computing skills and large hard-coded lookup-tables. As a result, we do not distinguish fixed base (point) operations from unknown ones and accelerate them with fixed-window [44] uniformly by precomputing on the fly. Taking EC as an example, a point is defined as an object with a member multiples for storing the precomputed values. multiples will be filled during the first point multiplication performed on the object and then be cached for reuse.

Based on the above fundamental modules and other cryptographic primitives (e.g., SHA256 [20], PBKDF2 [28]) provided by $s j c l$, we implement client-side protocols in srp.js and spake2p.js, and then form into the library pake.js with necessary tools defined in utils.js. Finally, the modules are compressed into a 61-KB js file.

4.1.2 CUDA Implementation. An appropriate sampling routine for arbitrary-precision integers can significantly improve the overall performance of PAKE implementations. Although a large sampling size increases the degree of parallelism, the principle 
is under the restriction of the word size of the platform-specific fixed-precision number. To achieve the product of two words without losing precision, the word size tends to be less than half of a machine word. In this work, we adopt a state-of-the-art data representation proposed by N. Emmart et. al. [10] for $G F(p)$ operations on GPUs.

Emmart et. al.'s method: The method samples a large integer in 52-bit limbs, with each limb stored as the mantissa of a double-precision floating-point (DPF) number which is denoted as double in most programming languages. Compared with other variable types, the floating-point format and its arithmetic are standardized in IEEE 754 [19], thus behavior diversities of DPF are eliminated on all standard-compliant platforms. With the help of fused-multiply-and-add (FMA) instruction, such sampling strategy conducts a full product of two 52-bit limbs efficiently as shown in Figure 6. The involvement of $\mathrm{c} 1$ and $\mathrm{c} 2$ could align the valid bits of hi and lo, thus avoid possible overflows in later accumulation. With Montgomery reduction implementation based on the above idea and some additional tricks, the scheme outperforms all of the alternatives proposed for modular exponentiation on GPUs before.

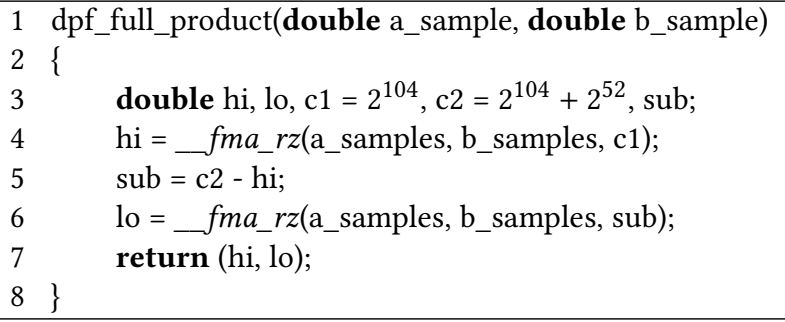

Figure 6: dpf_full_product for GPUs

For the underlying Edwards25519 curve of SPAKE2+ protocol, we use Gao et. al.'s method (called DPF-ECC) [11] which applies Emmart et. al.'s scheme for fast reduction over $G F(p 25519)$ with ingenious modifications.

DPF-ECC: The method tweaks Emmart et. al.'s scheme to a mixed-radix representation, sampling a 256-bit number into four 51-bit limbs and a 52-bit limb (also the most significant limb). To keep the alignment of the product of two limbs with unfixed word size, variables $\mathrm{c} 1$ and $\mathrm{c} 2$ in function dpf_full_product of Figure 13 are set to $2^{103}$ and $2^{103}+2^{52}$. Since the modulus, $p 25519$ is a pseudo-Mersenne prime which allows fast reduction, outputs of underlying addition and subtraction over $G F(p 25519)$ converge to the range $\left[0,2^{255}+4845\right)$ with only one round of reduction.

As registers are precious resources in GPUs, we split an operand into multiple pieces according to the modulus length of $G F(p)$ and perform operations with the corresponding number of threads in parallel. In contrast, an element of $G F(p 25519)$ consists of only five limbs, and thus no longer needs threads co-operation.

4.1.3 AVX-512 Implementation. AVX-512 provides FMA instructions including $v$ fmadd $132 p d$, $v$ fmadd213pd and $v$ fmadd231pd, making it convenient to transplant Emmart et. al.'s algorithm to CPU. While AVX-512 broadcasts the same instruction to each element of the vector, it multiplies a 52-bit limb $b$ by $a$ with eight 52-bit limbs. As a result, we implement vectorized full product as shown in Figure 13.

Actually, AVX-512 offers an IFMA feature that performs FMA on 52-bit integers. With this new feature, the function in Figure 13 could be implemented simply and elegantly, as shown in Figure 14.
However, IFMA has not been supported by any desktop processor yet.

When it comes to $G F(p 25519)$, the best sampling strategy seems to be 32-bit or 64-bit limbs, and therefore, a ZMM register could neatly hold two Edwards 25519 coordinates. In addition, underlying finite field operations of extended coordinates tend to appear in pairs and are independent of each other. However, some requisite instructions, e.g., vpadcd, vpsbbd, vpmulld, vpmulhud, are only available on Intel phi processors which are somehow obsolete comparing to GPUs for the sake of low frequency, high TDP, limited resources and rigorous requirements for hardware platforms. Based on the above fact, we still apply $D P F-E C C$ scheme for AVX implementation.

However, DPF-ECC cannot be smoothly transplanted to vector instructions, since the mixed-radix representation interrupts the unity of operations on the elements of a vector variable. We preset some important vectors in Table 10 for later computations, with the $i$-th element of a vectorized variable (i.e. _ m512i, _. m512d) $a$ denoted as $a[i]$ (just like an array) for convenience.

Pre-computation: The square-and-multiply approach for exponentiation (i.e. $b^{e}$ ) takes $l$ times of squaring and multiplications where $l$ is the bit length of the group modulus. We exploit $w$-bit fixed-window to accelerate unknown base modular exponentiation, taking $l$ times of squaring, $l / w$ times of memoryaccess-and-multiply, and extra $2^{w}-2$ multiplications for precomputation. When it comes to fixed bases, a much larger table could be computed offline. With $j$-th power of $b^{i \times w}$ stored as $\operatorname{pret} b[i][j]$ where $0<=i<\lceil l / w\rceil$ and $0<=j<2^{w}$. After that $b^{e}$ is calculated as $\prod_{i=0}^{l / w-1}$ pretb $[i]\left[e_{i \times w:(i+1) \times w-1}\right]$. We store the elements of pretb as double type for convenience and the offline file's size $M=2^{w} \times\lceil l / 52\rceil \times\lceil l / w\rceil \times 64$ bits.

To accelerate known-point multiplication on EC groups, we pre-compute offline table as depicted in [22] with window size 16. In addition, we compress the coordinates as defined in RFC7748 to save the storage. As a result, the offline file's size is $\lceil 255 / 16\rceil \times$ $32 \times 2{ }^{16} B=32 M B$. As for unknown point multiplication, we use the 4-bit fixed window method for side-channel considerations.

Carry Resolve and Reductions: It is noticeable that $D P F-$ $E C C$ brings additional overheads for reductions when implemented with vector instructions for the disunity of its word size. Standard carry resolution approaches are no longer applicable to AVX-based $G F(p 25519)$ elements and thus, we exploit carrypredicting technique [9] for finite field operations.

Figure 15 gives the vectorized modular addition with carrypredicting as example. Note that the last carry resolve from Step 17 to Step 21 is slightly different from the standard carry-predicting at the beginning (Line 6 to Line 9). Since the fast reduction in step 15 adds a little number (less than 39) to $c[0]$, bringing no carry immediately to $c[1 \sim 4]$. As a result, we could obtain the information about generate as well as propagate at once by comparing $c$ with the special mask $\left[2^{51}, 2^{51}-1,2^{51}-1,2^{51}-1,2^{52}-1,0,0,0\right]$. Specifically, the least significant bit of generate indicates the only possible carry bit and the left ones imply propagation of $a[1 \sim 4]$.

In traditional ECC-based applications such as digital signature, partially reduced coordinates won't affect the correctness. However, this view does not hold for PAKE protocols in which a coordinate may be input to hash functions. In other words, two secrets congruent modulo $q$, i.e. $s_{1}=s_{2} \bmod q$ while $s_{1} \neq s_{2}$, would lead to distinct session keys. 
There are two intermediate result forms not thoroughly reduced in $G F(p 25519)$ operations under the lazy-reduction strategy:

- Case 1: $2^{255}<=a<2^{255}+4845$, more specifically, $a[4]=$ $2^{51}, a[1]=a[2]=a[3]=0$ and $a[0]<4845 . a$ converges to $[0,4864)$ after a fast reduction without generating any carry bit.

- Case 2: $2^{255}-19<=a<2^{255}$, with the elements satisfying $a[1]=a[2]=a[3]=a[4]=2^{51}-1$ and $2^{51}-19<=a[0]<$ $2^{51}$. Under this case $a$ is required to subtract $q$ to get fully reduced.

Since the above two cases never occur at the same time, we perform both the two reductions for side-channel resistance as shown in Figure 16.

\subsection{Protocol Stack}

Based on the primitives introduced above, we construct a protocol stack including SRP and SPAKE2+ across client-side and serverside.

4.2.1 Client Side. As we have discussed before, offline computing is infeasible on the client-side because of the mechanism of JavaScript. Initial vectors and look-up tables will be generated on instantiating of the owner object. The pre-computed table of a number or an EC point in pake.js is generated on the fly and then cached for reused in subsequent exponentiation or point multiplications. We make the group generator a member of the relevant object, i.e. $s r p$ and spake2p, hereafter the table takes effect in later authentications until the object is destroyed. According to Figure 1 , the immediate value $g^{x}$ in step 5 can also be reused, if we make it a member likewise.

4.2.2 Server Side. RFC5054 specifies prime groups with the representative modulus of 1024-bits to 8192-bits in length for SRP, and in this work, we adopt the first three whose modulus bit lengths are 1024, 1536 and 2048 respectively. According to Table 1, there are three modular exponentiation operations on the serverside during a single execution of SRP, including a fixed-base one. For an unknown base, we pre-compute the fixed window table for its exponentiation and determine the optimal window size as 6 after repeated trials for the three groups. For a fixed base such as a group generator, we use pre-computed tables for acceleration and set the window size $w$ to 8 . Consequently, the offline files are $5 \mathrm{MB}, 11.25 \mathrm{MB}$ and $20 \mathrm{MB}$ respectively. A slightly larger $w$ brings limited performance improvement but an exponential increase of memory occupation. However, if $w$ doubles, the memory footprint will reach $640 \mathrm{MB}, 1440 \mathrm{MB}$ and $2560 \mathrm{MB}$, which are unacceptable for either RAM or global memory on GPUs. For large integers of the three lengths, we conduct calculations with $\lfloor l / 1024\rfloor \times 4$ threads on GPUs through trials, where $l$ is the modulus length in bits of a designated group, that is, 4 threads for 1024-bits and 1536-bits while 8 threads for 2048-bits.

The verifier of SPAKE2 + consists of $w_{0}$ and $L=\left[w_{1}\right] P$, where $w_{0}$ and $w_{1}$ are elements of $G F(p 25519)$ derived from user's password and $P$ is a generator of Edwards25519 group. In our framework, the Web server sends tuple $\left(w_{0}, L, X\right)$ to PAKE-Server and gets a response with $(Y, Z, V)$. As $M, N$ and $w_{0}$ are fixed for an individual user, we compute the point multiplications $\left[w_{0}\right] N$ and $\left[w_{0}\right] M$ in Step 5 and Step 6 of Figure 2 in advance and replace the original verifier $\left(w_{0}, L\right)$ in database with $\left(w_{0},\left[w_{0}\right] M,\left[w_{0}\right] N, L\right)$. Consequently, the tuple sent to PAKE-Server becomes ([ $\left.w_{0}\right] M$, $\left.\left[w_{0}\right] N, L, X\right)$ with 32 -bytes larger in size than before, and this modification saves two point multiplications at the expense of a 32-bytes increase in the size of each request to PAKE-Server and additional 4 point-decompresses which contain square roots.

\subsection{Up-level Optimizations}

While the optimized protocol stack helps speed up the execution of a single PAKE instance, reasonable I/O handling and task scheduling will contribute to the overall performance of the system. Figure 7 shows the detailed structure of PAKE-Server.

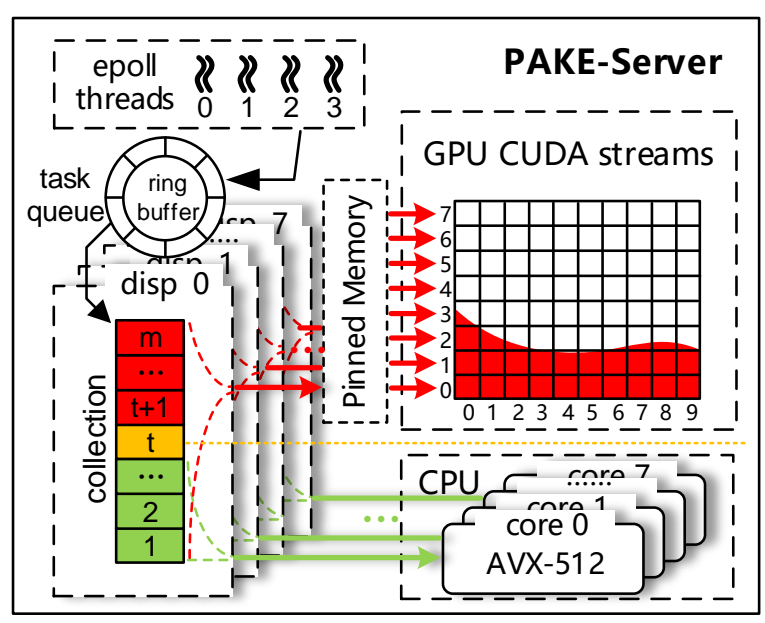

Figure 7: Detailed Structure of PAKE-Server

4.3.1 Asynchronous Computing Routine. While aPAKE service belongs to "computation-bound" programs for its heavy overheads, we adopt an asynchronous way to avoid I/O blocking under highly concurrent requests. More specifically, requests are not processed immediately on arrival, instead, they are collected by a group of dispatching threads for batch processing. This method is widely used in GPU-accelerated servers, where at least a global thread is required for gathering up tasks. However, most of the time the tasks are too little to fully leverage the computing power of GPUs and consequently, an individual request's latency is higher than CPU-based implementations. This is why we provide AVX-accelerated implementations in addition to CUDA programs. The system contains an I/O module and a worker module.

4.3.2 I/O Module. This module consists of multiple epoll threads and dispatchers bridged by a task queue. As a new system call introduced in Linux 2.5.44 to replace the older POSIX select [26] and poll [25], epoll [24] solves the problem of scalable I/O event notification. An epoll thread monitors a socket pool with epoll interfaces, sending the IDs of arrived packages to the task queue. A dispatcher (denoted by disp in the figure) collects tasks from the queue and routes them to CPU or GPUs according to the task number and dispatching strategy. The two types of threads are not the more the better. Besides the restriction of CPU resources, too many dispatchers also lead to inefficient GPU utilization. The processor we use has 10 physical cores, which means 20 logical cores with hyper-threading enabled. Owing to the excellent behavior of epoll, we only keep 4 epoll threads and 2 task queues. The dispatcher number is set to 8 over repeated trials. As our device is equipped with an NVIDIA Titan V card which has 80 streaming multiprocessors (sm), we maintain 10 CUDA streams 
for each dispatcher to fully exploit the parallelism of GPUs. With the zero-copy feature enabled since CUDA 2.2, we allocate pinned mapped memory for each stream in advance to avoid expensive host-device memory copy.

4.3.3 Worker Module. Tasks are forwarded to this module for computation finally. For each protocol, we provide two implementation versions, host program based on AVX-512 instructions and CUDA program for GPUs. With the control of dispatchers, we construct a heterogeneous computing model. If the request number is "relatively small", the tasks will be finished by the host program one by one. Otherwise, the tasks will be launched to GPUs and processed by the CUDA program in bulk. According to the specifications of our graphics card listed in Table 2, we schedule the CUDA threads as follows:

- BlocksPerGrid $=2$

- GridsPerStream = MaxResidentBlocksPerSM / BlocksPerGrid $=16$

- ThreadsPerBlock = MaxResidentThreadsPerSM / MaxResidentBlocksPerSM = 64

Table 2: Specifications of NVIDIA Titan V (Compute Capability 7.0)

\begin{tabular}{c|c}
\hline Number of Streaming Multiprocessors (SM) & 80 \\
\hline Max Resident Threads Per SM & 2048 \\
\hline Max Resident Blocks Per SM & 32 \\
\hline Max Resident Grids Per Device & 128 \\
\hline
\end{tabular}

There ought to be a threshold $t$ in a dispatcher to judge whether the tasks should be sent to GPUs or not. The proper value of $t$ depends on multiple factors, such as computational overheads, optimizations, hardware specifications, etc. In other words, $t$ is an empirical value, and we will determine the optimal $t$ of each protocol implementation through experiments in Section 5.

\subsection{Application to Web Systems}

The overall framework of our scheme is displayed in Figure 8. The dotted box at the left indicates the border of the Web application, with the blue shading blocks highlighting new components for vendors to integrate.

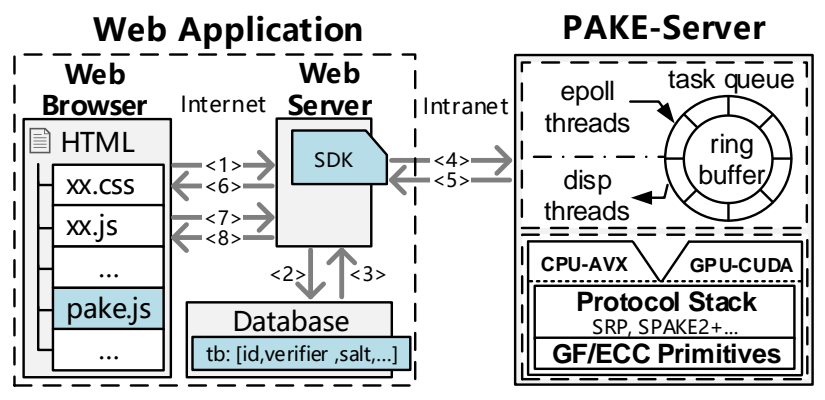

Figure 8: Overall Framework

For Web browsers, vendors only need to insert pake.js into their front-end code. Meanwhile, PAKE-Server deployed in Intranet takes over the most costly work of aPAKE computations from Web servers.

In contrast, upgrading Web servers is a bit more complicated. The most troublesome job is database modification. If the target protocol (e.g., SRP) hashes the password together with a random salt that the system hasn't used before, the developers should establish storage for salts. While vendors are suggested to store the salts separately from the passwords in case of leakage, the involvement of salt won't change the structure of a table. The functionalities of server-side aPAKE could be divided into two categories, lightweight operations (e.g., hash, KDF, HMAC), and resource-intensive public-key computations. Since the latter type of operations is highly centralized and computationally expensive, they are offloaded to the PAKE-Server. Web servers are required to integrate the left functions and TCP connection management. In this work, we provide the new functionalities with an easy-to-integrate Software Development Kit (SDK), which acts as middleware.

It is worth mentioning that if the Web servers are equipped with AVX-512-available processors (such as Intel Xeon family based on Skylake microarchitecture), our scheme could be tweaked to a partial plug-in mode by transplanting CPU program to Web servers. Such adjustment further improves performance for minimal tasks.

The numbered arrows in Figure 8 indicate the data flow of authentication. Flow $\langle 1\rangle$ to $\langle 6\rangle$ derive the session key while the rest finish key confirmation. In our design, SRP and SPAKE2+ run as Table 3 shows.

Table 3: Transferred Parameters of SRP and SPAKE2+ in Figure 8

\begin{tabular}{c|c||c|c}
\hline & Data Flow & SRP & SPAKE2+ \\
\hline & $\langle 1\rangle$ & $I D, A$ & $I D, X$ \\
Key & $\langle 2\rangle$ & $($ select $s, v)$ & $\left(\right.$ select $\left.\left[w_{0}\right] M,\left[w_{0}\right] N, L\right)$ \\
Derivation & $\langle 3\rangle$ & $s, v$ & {$\left[w_{0}\right] M,\left[w_{0}\right] N, L$} \\
& $\langle 4\rangle$ & $A, v$ & $X,\left[w_{0}\right] M,\left[w_{0}\right] N, L$ \\
& $\langle 5\rangle$ & $u, B, S$ & $Y, Z, V$ \\
\hline Key & $\langle 6\rangle$ & $u, B, s$ & $Y$ \\
Confirmation & $\langle 7\rangle$ & $M_{1}$ & $K_{c A}$ \\
& $\langle 8\rangle$ & $M_{2}$ & $K_{c B}$ \\
\hline
\end{tabular}

\section{EVALUATION}

In this section, we conduct a series of experiments to reveal the performance of our scheme. The protocols we implement include SRP3 protocol with modulus size 1024-bit, 1536-bit and 2048-bit as well as SPAKE2+ protocol over Edwards25519 curve. For simplicity, we denote SRP implementation of supposed parameters with SRP- $l$, where $l$ is the modulus bit length, e.g. SRP-1024.

\subsection{Basic Configurations}

We build the testing environment under LAN as well as WLAN. Besides PAKE-Server and a Web server, the client devices include a wireless mobile phone and a PC connected to the network with a cable. Table 4 and Table 5 give the specifications and configurations of the devices.

Table 4: Server-side Specifications

\begin{tabular}{c||c|c}
\hline \multicolumn{1}{c||}{} & Web Server & PAKE-Server \\
\hline CPU & Intel $₫$ Xeon $\circledast$ E5-2697 v2 & Intel $\circledast$ Core(TM) i9-7900X \\
\hline GPU & - & NVIDIA GeForce GTX TITAN V \\
\hline RAM & 8GB & 16GB \\
\hline OS & Linux 4.15.0-64-generic, Ubuntu 16.04.16 & Linux 4.15.0-141-generic, Ubuntu 18.01 \\
\hline Network Card & 82574L Gigabit Network Connection & Ethernet Connection (2) I219-V \\
\hline Software & Apache/2.4.18 \& PHP 7.0.33 & - \\
\hline
\end{tabular}


Table 5: Clients' Specifications

\begin{tabular}{c||c|c}
\hline Product & Xiaomi Laptop Air 13 & HUAWEI Mate 30 Pro \\
\hline \hline CPU & Intel@ Core(TM) i5-8250U CPU @ 1.60GHz 1.80 GHz & HUAWEI Kirin 990 5G \\
\hline RAM & $8 \mathrm{~GB}$ & $8 \mathrm{~GB}$ \\
\hline OS & Windows 10 Family 20H2 & Android 10 \\
\hline
\end{tabular}

\subsection{Determining the Optimal Threshold}

As we have mentioned the threshold $t$ for heterogeneous computing in Section 4, we determine its optimal value through experiments here.

5.2.1 Theoretical Model. From the client-side's perspective, the total delay increases with the number of tasks sent to PAKEServer. Theoretically, the CPU program's delay increases linearly, conforming to $y=k \cdot x$ while the GPU program's delay increases stepwise, which could be concluded as $y=\left\lceil\frac{x}{B}\right\rceil \cdot T$. The variable $x$ denotes task number, and $y$ denotes delay. In addition, the parameter $T$ indicates the latency for a single task processed by GPUs, and $B$ implies the maximum amount of tasks that arrive simultaneously, which is decided by the connection number and the socket buffer's capacity for protocol-specific messages.

A proper $t$ shall be abscissa of the only intersection with the two function curves, and hence once the task number exceeds $t$ the GPU always finishes responds faster than the CPU. This requires the slope $k$ not to be less than $2 T / B$, and then the inequality $B>=\lceil 2 T / k\rceil$ holds. After that, we could get the optimal $t$ by substituting $y$ with $T$ in equation $y=k \cdot x$, and finally $t=T / k$.

Since $T$ could hardly be reduced, the feasible approaches to guarantee the existence and the uniqueness of $t$ include enlarging $B$ by expanding the socket buffer and establishing more connections to PAKE-Server. Our experiment intends to acquire $T$ and the lower limit of $B$ for each protocol.

5.2.2 Experiment Setup. Instead of sending requests from browsers, we launch tests on PAKE-Server directly with a client program that could simulate requests with configurable parameters like task amount, connection number. We only maintain one dispatcher for the test to avoid the error resulted from thread competitions.

The function curves of time-delay are shown in Figure 9 and more detailed data is given in Table 11 in Appendix B. Since we adopt a small socket buffer size (4096-bytes) for convenience, the two curves in each diagram do not intersect at an expected point with a small $B$. However, this won't impact the accuracy of $t$.

Just as we analyzed above, in each subfigure, the slope $k$ of the CPU curve and the step height $T$ of the GPU curve are determined by the complexity of the target protocol. A heavier computation overhead leads to greater $k$ and $T$, and thus the four protocols could be sorted by complexity as SRP-2048, SRP-1536, SRP-1024 and SPAKE2+. After the statistical analysis for the experimental result, key parameters of each protocol are given in Table 6 . The row deduced $t$ means that the value is deduced as $t=\lceil T / k\rceil$, and empirical $t$ indicates the task number of the request whose response time firstly exceeds $T$ in Table 11 . The coincidence of the two rows implies the theoretical model's correctness.

\subsection{Performance Evaluation from Server's Perspective}

While multiple metrics are taken into account for performance evaluation of the system, particular concerns are paid to throughput

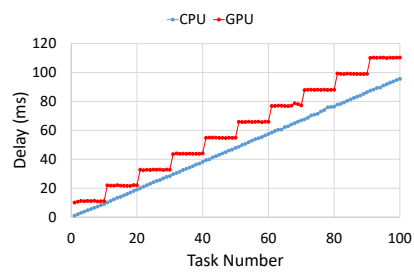

(a) SRP-1024

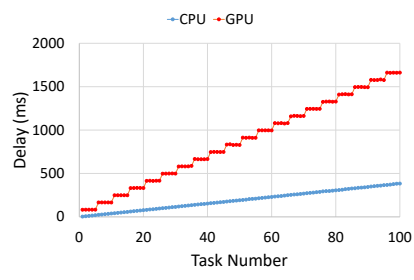

(c) SRP-2048

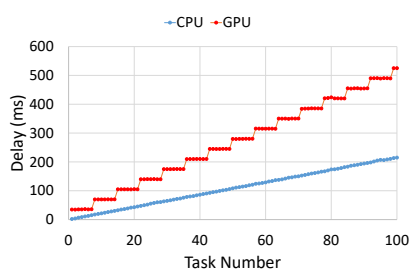

(b) SRP-1536

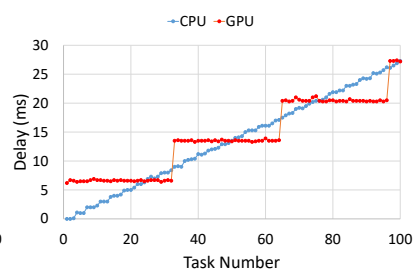

(d) SPAKE2+
Figure 9: Time-delay of a Single Dispatcher

Table 6: Key Parameters

\begin{tabular}{|c||c|c|c|c|}
\hline Protocol & SRP-1024 & SRP-1536 & SRP-2048 & SPAKE2+ \\
\hline \hline$T$ & 10.997 & 33.526 & 83.074 & 6.788 \\
\hline$k$ & 0.957 & 2.161 & 3.842 & 0.269 \\
\hline suggested $B(\lceil 2 T / k\rceil)$ & $\geq 23$ & $\geq 32$ & $\geq 44$ & $\geq 51$ \\
\hline deduced $t(\lceil T / k\rceil)$ & 12 & 16 & 22 & 26 \\
\hline empirical $t$ & 12 & 16 & 22 & 26 \\
\hline
\end{tabular}

$T$ : latency of a single task on GPUs $k$ : the slope of CPU delay function

$B$ : max number of tasks a socket buffer contains $t$ : threshold

for PAKE-Server, which indicates the tasks processed per second. By testing the maximize throughput, we expect to provide references for making the most of PAKE-Server's computing capacity.

5.3.1 Trade-off Considerations. The basic routine for improving throughput is to increase $c$ (number of connections to PAKEServer) and $p$ (number of tasks carried in a roundtrip message) by establishing more TCP connections with a larger socket buffer. However, larger $c$ or $p$ leads to higher resource consumption and logical complexity. A proper configuration for $c$ and $p$ helps the Web system harvest the best performance without obvious waste of resources. While the $p$ value of a protocol is determined after compilation, the $c$ value could be controlled by the vendors according to the scale of their server cluster. In our design, vendors are suggested to

(1) select the proper socket buffer size according to the current size and the probable size in the future of their server cluster, and

(2) establish an appropriate number of TCP connections to PAKE-Server in each Web server to ensure that it can achieve the ultimate throughput exactly.

5.3.2 Experiment Setup. In order to provide reasonable $c$ and $p$ values for vendors, we mount stress testing with different connection numbers and socket buffers size of 8192-bytes, 16384-bytes, 32768-bytes and 65536-bytes respectively. The detailed results are shown in Table 12 and Table 13 while intuitive results are drawn in Figure 10.

With $p$ determined by the socket buffer size, throughput increases with $c$ until the peak value. Vendors should ensure that 


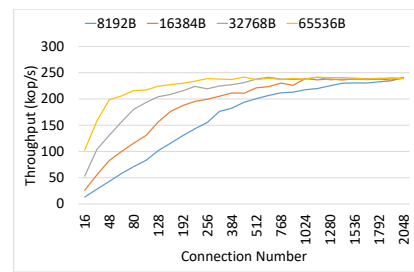

(a) SRP-1024

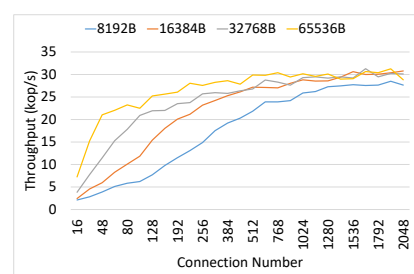

(c) SRP-2048

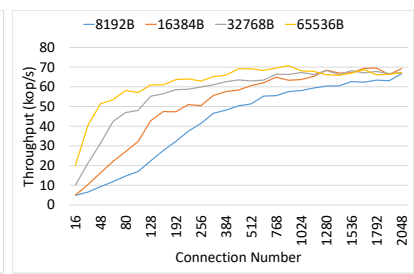

(b) SRP-1536

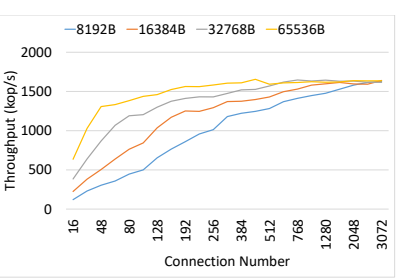

(d) SPAKE2+
Figure 10: Throughput of PAKE-Server

$c$ reaches the inflection point exactly in the figures. Oversized $c$ will not bring any performance improvement, instead, it leads to resource wastes of Web servers. Taking SPAKE2+ as an example, suppose that the Web server cluster contains 64 servers. According to Table 11, by adopting a 65536-bytes socket buffer, PAKE-Server could get fully loaded with only 5 TCP connections to each Web server. As long as the cluster gets expanded to no more than 320 servers in the future, vendors could maintain the best performance of the system by reducing TCP connections in each Web server to a proper number.

The abscissa of the inflection point of each curve in the figures represents the optimal number of connections under the current buffer size. Table 7 gives the maximum throughput of each protocol and the recommended $c$ value for each socket buffer size is listed in Table 8.

Table 7: Peak Throughput

\begin{tabular}{|c||c|c|c|c|}
\hline Protocol & SRP-1024 & SRP-1536 & SRP-2048 & SPAKE2+ \\
\hline \hline Peak Throughput (kop/s) & 241.536 & 70.740 & 30.155 & 1654.947 \\
\hline
\end{tabular}

Table 8: Suggested Connection Number

\begin{tabular}{|c||c|c|c|c|}
\hline \multicolumn{1}{|c||}{$\begin{array}{c}\text { Socket } \\
\text { Buffer Size }\end{array}$} & \multicolumn{4}{c|}{ Protocol } \\
\cline { 2 - 5 } & SRP-1024 & SRP-1536 & SRP-2048 & SPAKE2+ \\
\hline \hline 8192B & 2048 & 2048 & 2560 & 2560 \\
\hline 16384B & 1024 & 1152 & 1408 & 1280 \\
\hline 32768B & 512 & 768 & 1024 & 640 \\
\hline 65536B & 256 & 320 & 512 & 320 \\
\hline
\end{tabular}

\subsection{Performance Evaluation from Clients' Perspective}

In this subsection, we conduct tests for PAKE protocols from the client side. In comparison with PAKE-Server, we focus on user experience with the metric latency which implies the time delay from the user submitting the form to the moment session key is established.
5.4.1 Preparation. We set up a Web server with a simple PHP website on Apache server, and launch requests from four mainstream PC browsers and two mobile browsers. The detailed information of the browsers is listed in Table 9. Our implementations conform to RFC2945 and CFRG document [42], and the KDF function used for SPAKE2+ is PBKDF2 with iteration set to 1000 .

Table 9: Versions of Testing Platforms

\begin{tabular}{c|c}
\hline Platform & Version \\
\hline \hline Chrome & 90.0 .4430 .93 (Official Build) (64-bit) \\
Firefox & 88.0 (64-bit) \\
IE & 11 \\
Microsoft Edge & 90.0 .818 .51 (64-bit) \\
\hline Huawei Browser & 11.0 .8 .301 \\
Firefox for Android & 88.13 (Build \#2015808649) \\
\hline
\end{tabular}

5.4.2 Experiment Setup. In order to observe the detailed time consumption on the call path, we collect timestamps of several key nodes as shown in Figure 11, and then get the delays of desired phases.

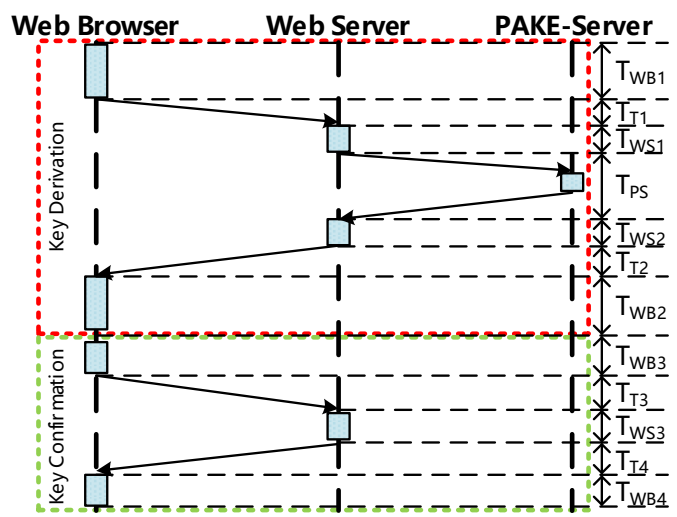

Figure 11: Time Slots, the subscript " $W B *$ " means "Web Browser", " $T *$ " means "Transmission", "WS*" means "Web Server", and "PS" means "PAKE-Server"

We launch tests during idle-hours as well as busy-hours of PAKE-Server. Considering the disturbance caused by the unstable network (especially WLAN), we sample the transmission delay $T_{T 1}, T_{T 2}, T_{T 3}$ and $T_{T 4}$ to diminish the infect of network variations. Influenced by the out-sync clocks between the client and the server, a single transmission delay is inaccurate (sometimes even be negative). However, the sum of them can offset this error, and we denote it with $T_{T}$. The testing results are shown in Table 14-17 in Appendix B.

To reveal the performance gap between PAKE schemes and a pure hash-based authentication, we test the latter's delay and list the results in Table 18. The hash function we adopted is Bcrypt. Figure 12 compares the latency during idle hours on the testing platforms with histograms. Although the hash-based approach seems to be much faster than PAKE schemes, its workloads are centralized in Web servers, leading to a rapid increase of latency with the concurrency up.

Unsurprisingly, the outdated kernel of IE results in the highest latency among the testing platforms. All the protocols could be finished within 3 seconds except for SRP-2048 on IE. Influenced 


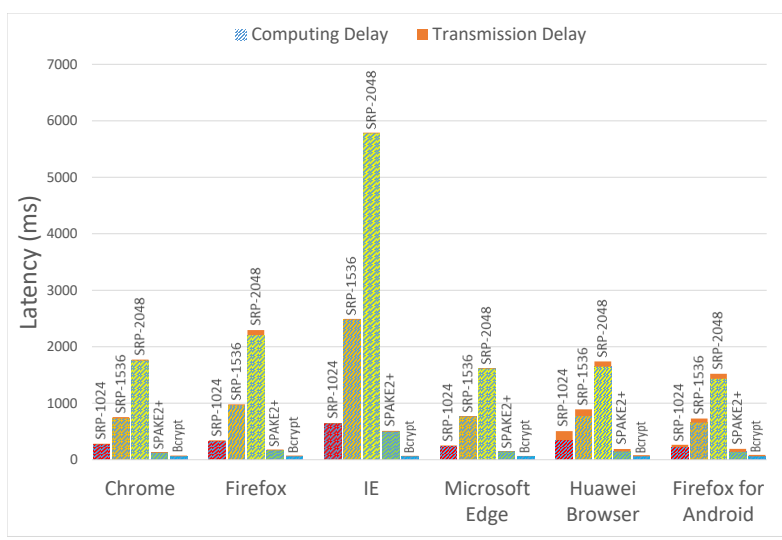

Figure 12: Latency during Idle-hours

by the natural shortage of wireless networks, the two mobile browsers receive higher transmission delays than the desktop ones. While a PAKE scheme achieves different performances on various browsers, the hash-based authentication obtains a stable delay since the hash operation is conducted on the server-side.

5.4.3 Result Analysis. In the low concurrency scenes, $T_{P S}$ only shares a small proportion (seldom exceeds $1 \%$ ) of the entire process. On the contrary, most time consumption occurs in Web browsers for the natural performance shortcomings of JavaScript and consequently, other factors including browser kernel, crypto parameters (such as the iteration number of PBKDF2) also impact the client-side's performance significantly, and thus latency of the same protocol varies on different platforms. When PAKE-Server is fully loaded, the user could acutely feel the increase of delay. According to the result, $T_{P S}$ under the busy state is $10 \mathrm{x}$ to $100 \mathrm{x}$ of that during idle time. Although the latency of a PAKE authentication seems to be times of a hash-based one, the bottleneck lies in browsers instead of Web servers. According to Table 18 and $T_{W S} *$ in Table 14-17, our scheme takes over almost all the burdens of Web servers in comparison with traditional hash-based approach, and hence the system's throughput increases by a large margin. It is noticeable that SPAKE2+ keeps latency lower than $0.4 \mathrm{~s}$ even when facing up with over 1,600,000 requests per second on most platforms.

\section{CONCLUSION}

In this work, we propose Heterogeneous-PAKE, a practical Web framework for the application of PAKE protocols. Our scheme also gives the vendors instructions about upgrading existing Web systems for PAKE authentication. To dispel people's misgiving about the feasibility of PAKE schemes in a real-world scene caused by their heavy computational overheads, we provide high-speed implementations of two representative PAKE protocols, SRP and SPAKE2+. The system achieves comprehensive performance comparable with hash-based authentications by integrating state-of-the-art research results into a heterogeneous computing module supported by GPUs and CPU with vector instructions. For PAKE protocols, this work bridges the gap between their theoretical research and the real-world deployment and provides a reference for industrialization.

\section{ACKNOWLEDGMENTS}

This work was supported in part by the National Natural Science Foundation of China under Grant 61902392 and in part by CCFTencent Open Fund under Grant RAGR20210131.

\section{REFERENCES}

[1] 2019. Facebook stored hundreds of millions of passwords in plain text. https://www.theverge.com/2019/3/21/18275837/facebook-plain-textpassword-storage-hundreds-millions-users

[2] 2019. Google stored some passwords in plain text for fourteen years-Only affects some G Suite customers. Technical Report. https://www.theverge.com/2019/ 5/21/18634842/google-passwords-plain-text-g-suite-fourteen-years

[3] Michel Abdalla and David Pointcheval. 2005. Simple password-based encrypted key exchange protocols. In Cryptographers' track at the RSA conference. Springer, 191-208.

[4] Steven Michael Bellovin and Michael Merritt. 1992. Encrypted key exchange: Password-based protocols secure against dictionary attacks. (1992).

[5] F. Callegati, W. Cerroni, and M. Ramilli. 2009. Man-in-the-Middle Attack to the HTTPS Protocol. Man-in-the-Middle Attack to the HTTPS Protocol.

[6] Ran Canetti. 2001. Universally composable security: A new paradigm for cryptographic protocols. In Proceedings 42nd IEEE Symposium on Foundations of Computer Science. IEEE, 136-145.

[7] Marius Cornea. 2015. Intel AVX-512 instructions and their use in the implementation of math functions. Intel Corporation (2015).

[8] Jiankuo Dong, Fangyu Zheng, Juanjuan Cheng, Jingqiang Lin, Wuqiong Pan, and Ziyang Wang. 2018. Towards high-performance X25519/448 key agreement in general purpose GPUs. In 2018 IEEE Conference on Communications and Network Security (CNS). IEEE, 1-9.

[9] Jiankuo Dong, Fangyu Zheng, Niall Emmart, Jingqiang Lin, and Charles Weems. 2018. sDPF-RSA: Utilizing floating-point computing power of GPUs for massive digital signature computations. In 2018 IEEE International Parallel and Distributed Processing Symposium (IPDPS). IEEE, 599-609.

[10] Niall Emmart, Fangyu Zheng, and Charles Weems. 2018. Faster modular exponentiation using double precision floating point arithmetic on the GPU. In 2018 IEEE 25th Symposium on Computer Arithmetic (ARITH). IEEE, 130-137.

[11] Lili Gao, Fangyu Zheng, Niall Emmart, Jiankuo Dong, Jingqiang Lin, and Charles Weems. 2020. DPF-ECC: Accelerating Elliptic Curve Cryptography with Floating-Point Computing Power of GPUs. In 2020 IEEE International Parallel and Distributed Processing Symposium (IPDPS). IEEE, 494-504.

[12] Jeffrey Goldberg. 2017. Three layers of encryption keeps you safe when SSL/TLS fails. Technical Report. https://blog.1password.com/three-layers-ofencryption-keeps-you-safe-when-ssl/tls-fails/

[13] IEEE P1363 Working Group et al. 2003. Standard specifications for passwordbased public-key cryptographic techniques. IEEE P1363. 2/D11 (2003).

[14] Huseyin Hisil, Kenneth Koon-Ho Wong, Gary Carter, and Ed Dawson. 2008. Twisted Edwards curves revisited. In International Conference on the Theory and Application of Cryptology and Information Security. Springer, 326-343.

[15] Apple Inc. 2021. Apple Platform Security. Technical Report. https://manuals.info.apple.com/MANUALS/1000/MA1902/en_US/appleplatform-security-guide.pdf

[16] D. JABLON. 1999. B-SPEKE. Integrity Sciences White Paper (1999). https: //ci.nii.ac.jp/naid/10010452557/en/

[17] Stanislaw Jarecki, Hugo Krawczyk, and Jiayu Xu. 2018. OPAQUE: an asymmetric PAKE protocol secure against pre-computation attacks. In Annual International Conference on the Theory and Applications of Cryptographic Techniques. Springer, 456-486.

[18] Hwancheol Jeong, Sunghoon Kim, Weonjong Lee, and Seok-Ho Myung. 2012. Performance of SSE and AVX instruction sets. arXiv preprint arXiv:1211.0820 (2012).

[19] William Kahan. 1996. IEEE standard 754 for binary floating-point arithmetic. Lecture Notes on the Status of IEEE 754, 94720-1776 (1996), 11.

[20] Cameron F Kerry and Charles Romine Director. 2013. FIPS PUB 186-4 federal information processing standards publication digital signature standard (DSS). (2013).

[21] Taekyoung Kwon. 2001. Authentication and Key Agreement via Memorable Password.. In NDSS.

[22] K. Leboeuf, R. Muscedere, and M. Ahmadi. 2013. A GPU implementation of the Montgomery multiplication algorithm for elliptic curve cryptography. IEEE (2013).

[23] Philip MacKenzie. 2002. The PAK suite: Protocols for password-authenticated key exchange. In IEEE P1363. 2. Citeseer.

[24] Michael Kerrisk. 2021. epoll(7) - Linux manual page. https://man7.org/linux/ man-pages/man7/epoll.7.html [Online; accessed 16-June-2021]

[25] Michael Kerrisk. 2021. poll(2) - Linux manual page. https://man7.org/linux/ man-pages/man2/poll.2.html [Online; accessed 16-June-2021]

[26] Michael Kerrisk. 2021. select(2) - Linux manual page. https://man7.org/linux/ man-pages/man2/select.2.html [Online; accessed 16-June-2021].

[27] Montgomery and L. Peter. 1985. Modular multiplication without trial division. Math. Comp. 44, 170 (1985), 519-519.

[28] Kathleen Moriarty, Burt Kaliski, and Andreas Rusch. 2017. Pkcs\# 5: Passwordbased cryptography specification version 2.1. Internet Eng. Task Force (IETF) 8018 (2017), 1-40. 
[29] Junghyun Nam, Juryon Paik, H-K Kang, Ung Mo Kim, and Dongho Won. 2009. An off-line dictionary attack on a simple three-party key exchange protocol. IEEE Communications Letters 13, 3 (2009), 205-207.

[30] Stuart Oberman, Greg Favor, and Fred Weber. 1999. AMD 3DNow! technology: Architecture and implementations. IEEE Micro 19, 2 (1999), 37-48.

[31] Wuqiong Pan, Fangyu Zheng, Yuan Zhao, Wen-Tao Zhu, and Jiwu Jing. 2016. An efficient elliptic curve cryptography signature server with GPU acceleration. IEEE Transactions on Information Forensics and Security 12, 1 (2016), $111-122$.

[32] Alex Peleg and Uri Weiser. 1996. MMX technology extension to the Intel architecture. IEEE micro 16, 4 (1996), 42-50.

[33] Colin Percival and Simon Josefsson. 2016. The scrypt password-based key derivation function. IETF Draft URL: http://tools. ietf. org/html/josefsson-scryptkdf-00. txt (accessed: 30.11. 2012) (2016).

[34] Mark Pilgrim. 2010. Dive into HTML5. URL: http://diveintohtml5.info/index.html (2010).

[35] Niels Provos and David Mazieres. 1999. Bcrypt algorithm. In USENIX.

[36] Venu Gopal Reddy. 2008. Neon technology introduction. ARM Corporation 4, 1 (2008).

[37] Eric Rescorla and Tim Dierks. 2018. The transport layer security (TLS) protocol version 1.3. (2018).

[38] Jason Sanders and Edward Kandrot. 2010. CUDA by example: an introduction to general-purpose GPU programming. Addison-Wesley Professional.

[39] Nicolas Serrano, Hilda Hadan, and L Jean Camp. 2019. A complete study of PKI (PKI's Known Incidents). Available at SSRN 3425554 (2019).

[40] SeongHan Shin and Kazukuni Kobara. 2012. Efficient Augmented PasswordOnly Authentication and Key Exchange for IKEv2. RFC 6628. https://doi.org/ 10.17487/RFC6628

[41] Emily Stark, Michael Hamburg, and Dan Boneh. 2009. Symmetric cryptography in javascript. In 2009 Annual Computer Security Applications Conference. IEEE, 373-381.

[42] Tim Taubert and Christopher A. Wood. 2020. SPAKE2+, an Augmented PAKE. https://datatracker.ietf.org/doc/html/draft-bar-cfrg-spake2plus-02 Work in Progress.

[43] Abi Tyas Tunggal. 2021. The 56 Biggest Data Breaches (Updated for 2021). Technical Report. https://www.upguard.com/blog/biggest-data-breaches

[44] Henk C. A. van Tilborg and Sushil Jajodia (Eds.). 2011. Fixed Window Exponentiation. Springer US, Boston, MA, 482-482. https://doi.org/10.1007/978-14419-5906-5_1168

[45] Y. Wang. 2001. IEEE P1363.2 Submission / D2001-06-21,. P1363.2-ecsrp-0621.doc. (21 June 2001)

[46] Thomas Wu. 2002. Srp-6: Improvements and refinements to the secure remote password protocol. http://srp. stanford. edu/srp6. ps (2002).

[47] Thomas D Wu et al. 1998. The Secure Remote Password Protocol.. In NDSS, Vol. 98. Citeseer, 97-111.

\section{A AVX-512 CODES}

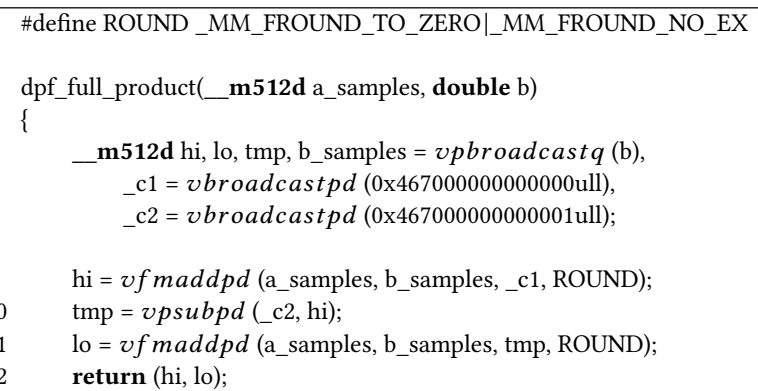

vproadcastq is abbreviated for sequence of instructions which broadcasts DPF (64-bit) $a$ to all elements of $d s t$

$v f$ maddpd here denotes the combination of $v$ fmadd132pd,

vf madd $213 p d$ and $v$ fmadd $231 p d$

Figure 13: dpf_full_product for AVX-512

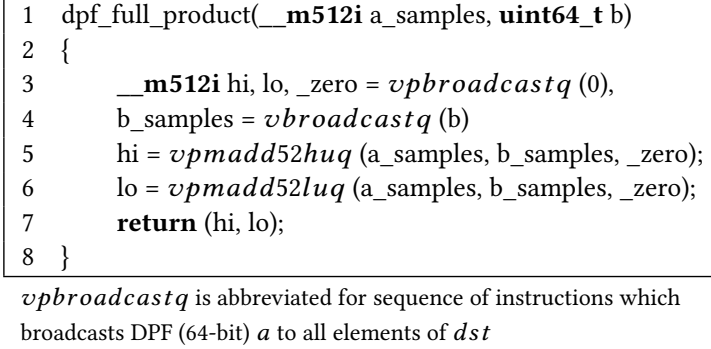

Figure 14: dpf_full_product for AVX-512 with IFMA

Table 10: Preseted Variables

\begin{tabular}{c|l}
\hline Symbol & ZMM Elements (LE) \\
\hline ones & {$[1,1,1,1,1,1,1,1]$} \\
$\_$19 & {$[19,19,19,19,19,19,19,19]$} \\
_q & {$\left[2^{51}-19,2^{51}-1,2^{51}-1,2^{51}-1,2^{51}-1,0,0,0\right]$} \\
mask51 & {$\left[2^{51}-1,2^{51}-1,2^{51}-1,2^{51}-1,2^{51}-1,2^{51}-1,2^{51}-1,2^{51}-1\right]$} \\
mask & {$\left[2^{51}-1,2^{51}-1,2^{51}-1,2^{51}-1,2^{51}-1,2^{51}-1,2^{51}-1,2^{52}-1\right]$} \\
\hline
\end{tabular}

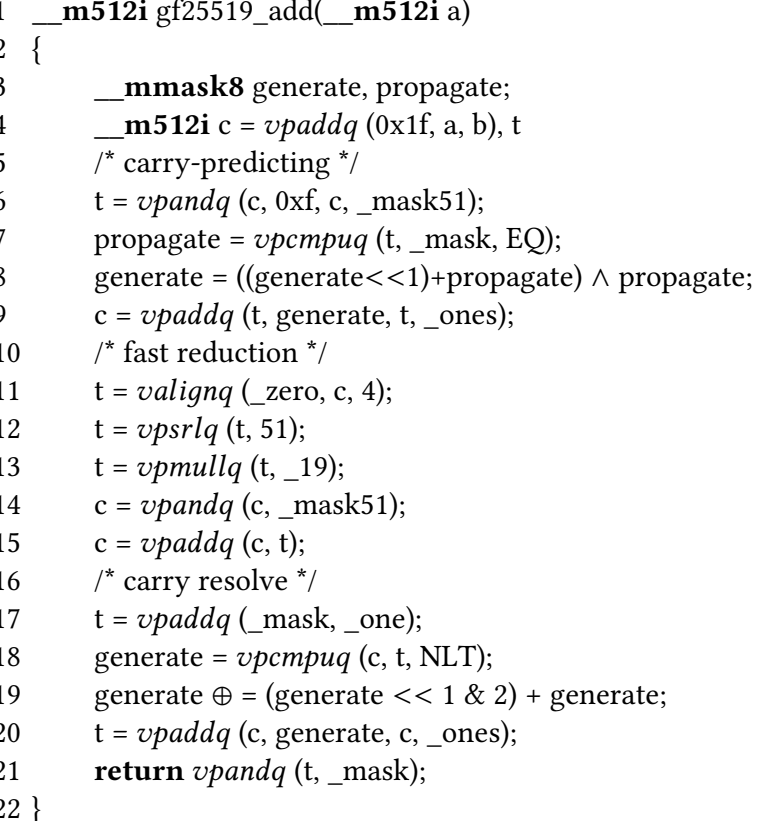

Figure 15: Modular Addition over $G F(p 25519)$

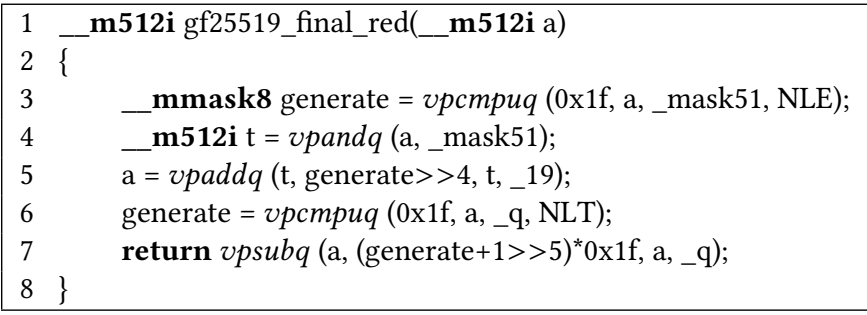

Figure 16: Final Reduction over $G F(p 25519)$ 
Table 11: Time-delay of a Single Dispatcher

\begin{tabular}{|c|c|c|c|c|c|c|c|c|c|c|c|c|c|c|c|c|c|}
\hline \multirow{2}{*}{ Tasks } & \multicolumn{2}{|c|}{ SRP-1024 } & \multicolumn{2}{|c|}{ SRP-1536 } & \multicolumn{2}{|c|}{ SRP-2048 } & \multicolumn{2}{|c|}{ SPAKE2+ } & \multirow{2}{*}{ Tasks } & \multicolumn{2}{|c|}{ SRP-1024 } & \multicolumn{2}{|c|}{ SRP-1536 } & \multicolumn{2}{|c|}{ SRP-2048 } & \multicolumn{2}{|c|}{ SPAKE2+ } \\
\hline & $\mathrm{CPU}$ & GPU & $\mathrm{CPU}$ & GPU & $\mathrm{CPU}$ & GPU & $\mathrm{CPU}$ & GPU & & $\mathrm{CPU}$ & GPU & $\mathrm{CPU}$ & GPU & $\mathrm{CPU}$ & GPU & $\mathrm{CPU}$ & GPU \\
\hline 1 & 1.0 & 10.1 & 2.0 & 35.0 & 3.8 & 82.4 & 0.0 & 6.2 & 51 & 48.7 & 65.9 & 111.0 & 279.5 & 195.3 & 913.4 & 14.0 & 13.6 \\
\hline 2 & 2.0 & 10.7 & 4.0 & 34.8 & 7.8 & 83.1 & 0.0 & 6.7 & 52 & 49.9 & 65.8 & 112.6 & 280.0 & 199.2 & 911.2 & 14.1 & 13.5 \\
\hline 3 & 3.0 & 11.2 & 6.8 & 35.3 & 11.7 & 82.9 & 0.1 & 6.6 & 53 & 50.7 & 65.8 & 114.5 & 280.0 & 205.5 & 913.9 & 14.3 & 13.5 \\
\hline 4 & 3.8 & 11.0 & 8.7 & 35.3 & 15.3 & 83.4 & 1.1 & 6.4 & 54 & 51.8 & 66.0 & 116.1 & 280.3 & 207.3 & 910.9 & 15.0 & 13.5 \\
\hline 5 & 4.8 & 11.2 & 11.0 & 36.3 & 19.6 & 83.0 & 1.0 & 6.5 & 55 & 52.9 & 65.8 & 119.1 & 280.1 & 210.2 & 911.2 & 15.3 & 13.5 \\
\hline 6 & 5.6 & 11.1 & 13.0 & 35.3 & 23.7 & 166.2 & 1.0 & 6.5 & 56 & 53.9 & 65.9 & 120.9 & 280.4 & 214.8 & 997.4 & 15.3 & 13.3 \\
\hline 7 & 6.5 & 11.3 & 15.2 & 35.8 & 27.5 & 166.2 & 2.0 & 6.5 & 57 & 54.6 & 66.0 & 124.3 & 315.6 & 218.8 & 997.5 & 15.3 & 13.4 \\
\hline 8 & 7.3 & 10.9 & 17.6 & 70.1 & 31.1 & 166.5 & 2.0 & 6.7 & 58 & 55.4 & 65.7 & 125.1 & 315.5 & 222.6 & 996.8 & 15.9 & 13.5 \\
\hline 9 & 8.3 & 11.0 & 19.7 & 70.0 & 34.9 & 166.2 & 2.0 & 6.9 & 59 & 56.5 & 66.0 & 127.1 & 315.3 & 226.5 & 996.9 & 16.1 & 13.5 \\
\hline 10 & 9.1 & 11.1 & 21.7 & 70.0 & 38.8 & 166.0 & 2.3 & 6.7 & 60 & 57.5 & 65.9 & 128.9 & 315.2 & 229.8 & 996.1 & 16.1 & 13.9 \\
\hline 11 & 10.2 & 22.0 & 23.8 & 70.0 & 42.5 & 249.2 & 3.0 & 6.7 & 61 & 58.4 & 76.9 & 132.1 & 315.5 & 233.9 & 1080.4 & 16.1 & 13.5 \\
\hline 12 & 11.2 & 21.8 & 26.1 & 70.2 & 46.4 & 249.4 & 3.0 & 6.6 & 62 & 59.6 & 76.9 & 133.8 & 315.5 & 237.5 & 1078.3 & 16.5 & 13.5 \\
\hline 13 & 12.3 & 21.8 & 28.4 & 70.0 & 49.8 & 249.5 & 3.0 & 6.6 & 63 & 60.5 & 77.1 & 136.9 & 315.0 & 240.6 & 1080.7 & 17.0 & 13.5 \\
\hline 14 & 13.4 & 22.1 & 30.2 & 70.3 & 54.0 & 249.5 & 3.8 & 6.5 & 64 & 60.6 & 77.0 & 137.8 & 350.3 & 245.1 & 1076.2 & 17.1 & 13.6 \\
\hline 15 & 14.0 & 21.8 & 32.4 & 105.0 & 57.5 & 249.3 & 4.0 & 6.7 & 65 & 62.3 & 76.9 & 139.5 & 350.1 & 249.9 & 1080.4 & 17.5 & 20.4 \\
\hline 16 & 15.0 & 21.7 & 35.0 & 105.1 & 62.0 & 332.0 & 4.0 & 6.6 & 66 & 62.8 & 76.8 & 142.2 & 350.3 & 253.8 & 1158.8 & 17.9 & 20.5 \\
\hline 17 & 16.0 & 21.6 & 36.6 & 105.1 & 65.8 & 332.3 & 4.2 & 6.7 & 67 & 64.1 & 77.1 & 145.3 & 349.4 & 258.2 & 1165.0 & 18.2 & 20.3 \\
\hline 18 & 17.0 & 21.6 & 38.8 & 105.0 & 69.4 & 334.3 & 4.9 & 6.6 & 68 & 65.0 & 78.7 & 146.6 & 350.5 & 260.2 & 1163.2 & 18.3 & 20.4 \\
\hline 19 & 18.1 & 22.2 & 41.5 & 104.9 & 73.7 & 333.6 & 5.0 & 6.6 & 69 & 66.0 & 78.1 & 149.1 & 350.2 & 264.4 & 1160.8 & 19.0 & 21.0 \\
\hline 20 & 19.0 & 22.0 & 43.0 & 105.5 & 77.8 & 332.3 & 5.0 & 6.6 & 70 & 66.9 & 77.3 & 150.0 & 350.6 & 268.2 & 1163.2 & 19.2 & 20.6 \\
\hline 21 & 20.0 & 32.8 & 45.4 & 105.1 & 81.0 & 415.4 & 5.4 & 6.5 & 71 & 67.6 & 87.9 & 152.6 & 384.2 & 272.6 & 1244.0 & 19.1 & 20.4 \\
\hline 22 & 21.1 & 32.5 & 47.5 & 140.0 & 85.3 & 416.8 & 6.0 & 6.6 & 72 & 68.7 & 88.1 & 154.8 & 385.0 & 276.9 & 1244.9 & 19.5 & 20.4 \\
\hline 23 & 22.1 & 32.8 & 49.9 & 140.0 & 88.2 & 414.9 & 6.0 & 6.7 & 73 & 70.3 & 88.1 & 157.4 & 385.1 & 280.1 & 1244.8 & 19.9 & 20.4 \\
\hline 24 & 23.1 & 32.6 & 52.0 & 140.3 & 92.6 & 417.3 & 6.3 & 6.5 & 74 & 70.9 & 88.0 & 159.7 & 385.8 & 284.9 & 1244.0 & 20.2 & 21.0 \\
\hline 25 & 24.1 & 32.9 & 55.2 & 140.2 & 96.5 & 417.3 & 6.9 & 6.6 & 75 & 71.5 & 88.1 & 161.4 & 385.6 & 287.7 & 1245.4 & 20.4 & 21.2 \\
\hline 26 & 25.0 & 32.8 & 57.7 & 140.5 & 100.2 & 498.3 & 7.3 & 6.7 & 76 & 73.1 & 88.0 & 163.7 & 385.5 & 293.9 & 1325.5 & 20.4 & 20.4 \\
\hline 27 & 25.7 & 32.9 & 60.1 & 140.0 & 103.7 & 499.1 & 7.0 & 6.7 & 77 & 74.1 & 88.1 & 166.3 & 385.3 & 297.1 & 1328.9 & 20.6 & 20.3 \\
\hline 28 & 26.8 & 32.6 & 60.7 & 139.8 & 107.8 & 499.3 & 7.3 & 6.7 & 78 & 75.9 & 88.0 & 167.3 & 420.7 & 298.2 & 1329.7 & 21.0 & 20.3 \\
\hline 29 & 27.8 & 32.9 & 63.1 & 175.0 & 111.9 & 500.1 & 7.9 & 6.4 & 79 & 76.2 & 88.0 & 170.4 & 421.7 & 301.8 & 1327.3 & 21.6 & 20.5 \\
\hline 30 & 28.3 & 32.8 & 65.0 & 174.9 & 115.0 & 498.7 & 8.0 & 6.6 & 80 & 76.4 & 88.1 & 174.0 & 423.9 & 306.0 & 1328.2 & 21.9 & 20.5 \\
\hline 31 & 29.7 & 43.6 & 66.9 & 174.9 & 119.6 & 580.9 & 8.0 & 6.7 & 81 & 77.8 & 99.3 & 174.6 & 420.4 & 309.2 & 1409.9 & 21.9 & 20.3 \\
\hline 32 & 30.5 & 44.1 & 69.4 & 175.3 & 123.6 & 582.2 & 8.4 & 6.6 & 82 & 78.3 & 99.1 & 176.5 & 420.5 & 313.2 & 1412.6 & 22.2 & 20.4 \\
\hline 33 & 31.4 & 43.8 & 71.7 & 175.5 & 127.0 & 581.6 & 9.0 & 13.5 & 83 & 79.3 & 99.0 & 178.8 & 420.1 & 319.3 & 1414.3 & 22.2 & 20.4 \\
\hline 34 & 32.6 & 43.9 & 73.1 & 175.1 & 130.8 & 581.9 & 9.1 & 13.6 & 84 & 80.4 & 99.2 & 181.9 & 420.1 & 322.6 & 1411.2 & 23.0 & 20.3 \\
\hline 35 & 33.4 & 43.8 & 76.0 & 175.2 & 135.0 & 588.1 & 9.0 & 13.5 & 85 & 81.4 & 99.1 & 183.5 & 455.2 & 326.9 & 1412.6 & 23.0 & 20.7 \\
\hline 36 & 34.3 & 43.9 & 78.6 & 209.9 & 138.7 & 667.2 & 10.0 & 13.5 & 86 & 82.4 & 99.1 & 186.7 & 454.4 & 329.4 & 1495.1 & 23.2 & 20.4 \\
\hline 37 & 35.2 & 43.8 & 80.3 & 210.1 & 142.9 & 664.4 & 10.2 & 13.5 & 87 & 83.3 & 99.0 & 188.5 & 455.2 & 335.0 & 1497.1 & 23.3 & 20.4 \\
\hline 38 & 36.4 & 43.8 & 81.7 & 210.0 & 146.7 & 664.3 & 10.3 & 13.6 & 88 & 84.1 & 99.0 & 190.1 & 455.4 & 337.8 & 1497.3 & 24.0 & 20.4 \\
\hline 39 & 37.0 & 43.8 & 84.3 & 210.3 & 149.6 & 664.0 & 10.4 & 13.3 & 89 & 85.3 & 99.0 & 192.5 & 454.4 & 340.2 & 1494.5 & 24.3 & 20.4 \\
\hline 40 & 38.2 & 44.1 & 86.4 & 210.3 & 153.4 & 666.1 & 11.2 & 13.5 & 90 & 86.4 & 99.1 & 194.5 & 454.7 & 344.7 & 1494.1 & 24.2 & 20.3 \\
\hline 41 & 39.4 & 54.8 & 88.7 & 210.1 & 157.7 & 746.9 & 11.1 & 13.5 & 91 & 87.4 & 110.1 & 196.4 & 455.2 & 350.0 & 1579.1 & 24.3 & 20.4 \\
\hline 42 & 39.9 & 55.0 & 90.6 & 210.3 & 161.4 & 749.2 & 11.3 & 13.5 & 92 & 88.2 & 110.3 & 198.1 & 490.0 & 354.3 & 1576.4 & 25.2 & 20.3 \\
\hline 43 & 41.3 & 55.0 & 92.8 & 245.2 & 165.2 & 748.4 & 11.8 & 13.6 & 93 & 89.3 & 110.2 & 201.6 & 490.2 & 357.7 & 1577.0 & 25.1 & 20.3 \\
\hline 44 & 42.1 & 55.0 & 95.2 & 245.2 & 168.9 & 747.7 & 12.0 & 13.4 & 94 & 89.6 & 110.3 & 204.8 & 490.5 & 360.6 & 1583.5 & 25.3 & 20.5 \\
\hline 45 & 43.1 & 54.8 & 97.0 & 244.9 & 172.4 & 748.7 & 12.1 & 13.6 & 95 & 91.0 & 110.3 & 207.2 & 489.2 & 365.8 & 1576.7 & 25.7 & 20.3 \\
\hline 46 & 44.1 & 54.8 & 99.5 & 244.9 & 176.7 & 833.3 & 12.3 & 13.4 & 96 & 91.9 & 110.0 & 206.2 & 490.6 & 367.9 & 1662.3 & 26.2 & 20.5 \\
\hline 47 & 44.9 & 54.7 & 101.6 & 245.4 & 181.1 & 836.9 & 12.9 & 13.7 & 97 & 93.0 & 110.3 & 208.6 & 490.3 & 371.8 & 1660.1 & 26.1 & 27.3 \\
\hline 48 & 46.1 & 54.9 & 103.5 & 245.4 & 184.6 & 828.6 & 12.9 & 13.5 & 98 & 93.8 & 110.2 & 210.6 & 489.7 & 377.3 & 1661.8 & 26.5 & 27.3 \\
\hline 49 & 46.7 & 54.8 & 105.8 & 245.1 & 187.6 & 831.2 & 13.1 & 13.5 & 99 & 94.8 & 110.3 & 213.3 & 525.2 & 381.9 & 1660.5 & 26.9 & 27.4 \\
\hline 50 & 47.9 & 54.9 & 108.5 & 279.7 & 192.0 & 828.5 & 13.5 & 13.4 & 100 & 95.6 & 110.3 & 214.9 & 525.2 & 383.9 & 1662.0 & 27.3 & 27.2 \\
\hline
\end{tabular}


Table 12: Throughput of a SRP (kops/s)

\begin{tabular}{|c|c|c|c|c|c|c|c|c|c|c|c|c|}
\hline \multirow{3}{*}{$\begin{array}{l}\text { Connection } \\
\text { Number }\end{array}$} & \multicolumn{4}{|c|}{ SRP-1024 } & \multicolumn{4}{|c|}{ SRP-1536 } & \multicolumn{4}{|c|}{ SRP-2048 } \\
\hline & \multicolumn{4}{|c|}{ Socket Buffer Size (B) } & \multicolumn{4}{|c|}{ Socket Buffer Size (B) } & \multicolumn{4}{|c|}{ Socket Buffer Size (B) } \\
\hline & 8192 & 16384 & 32768 & 65536 & 8192 & 16384 & 32768 & 65536 & 8192 & 16384 & 32768 & 65536 \\
\hline 16 & 13.110 & 26.041 & 53.043 & 102.469 & 4.844 & 4.988 & 10.087 & 20.163 & 2.116 & 2.433 & 3.832 & 7.245 \\
\hline 32 & 28.213 & 55.526 & 104.122 & 158.175 & 6.554 & 10.373 & 21.236 & 40.458 & 2.828 & 4.567 & 7.748 & 15.239 \\
\hline 48 & 42.896 & 82.644 & 130.609 & 198.172 & 9.279 & 16.303 & 31.318 & 51.423 & 3.898 & 5.948 & 11.462 & 21.036 \\
\hline 64 & 57.935 & 99.859 & 156.257 & 205.775 & 11.847 & 22.127 & 42.441 & 53.463 & 5.116 & 8.287 & 15.293 & 22.043 \\
\hline 80 & 71.050 & 115.669 & 180.343 & 215.866 & 14.663 & 27.112 & 46.935 & 58.104 & 5.840 & 10.083 & 17.814 & 23.241 \\
\hline 96 & 82.964 & 130.391 & 193.188 & 217.049 & 17.034 & 32.271 & 48.021 & 57.142 & 6.213 & 11.849 & 20.900 & 22.491 \\
\hline 128 & 101.578 & 156.214 & 204.262 & 224.135 & 22.367 & 42.776 & 55.245 & 60.979 & 7.704 & 15.414 & 21.934 & 25.228 \\
\hline 160 & 115.660 & 176.070 & 208.573 & 227.283 & 27.634 & 47.453 & 56.451 & 60.986 & 9.793 & 18.039 & 22.014 & 25.645 \\
\hline 192 & 130.041 & 187.414 & 215.543 & 229.813 & 32.250 & 47.312 & 58.553 & 63.641 & 11.512 & 20.076 & 23.521 & 26.081 \\
\hline 224 & 143.297 & 195.442 & 223.900 & 233.967 & 37.490 & 50.982 & 58.775 & 64.030 & 13.086 & 21.172 & 23.755 & 28.047 \\
\hline 256 & 155.111 & 199.325 & 219.218 & 238.710 & 41.380 & 50.443 & 59.906 & 62.923 & 14.875 & 23.190 & 25.699 & 27.574 \\
\hline 320 & 176.322 & 205.257 & 224.706 & 237.884 & 46.580 & 55.637 & 61.043 & 65.185 & 17.526 & 24.225 & 25.956 & 28.252 \\
\hline 384 & 182.363 & 211.364 & 227.113 & 237.109 & 48.132 & 57.561 & 62.574 & 65.903 & 19.201 & 25.294 & 25.796 & 28.626 \\
\hline 448 & 193.693 & 210.838 & 231.013 & 241.536 & 50.334 & 58.365 & 63.477 & 69.156 & 20.302 & 26.140 & 26.372 & 27.846 \\
\hline 512 & 200.456 & 221.170 & 237.861 & 236.871 & 51.329 & 60.596 & 62.970 & 69.156 & 21.843 & 27.175 & 26.775 & 29.886 \\
\hline 640 & 206.560 & 223.406 & 241.356 & 239.210 & 55.256 & 62.064 & 63.410 & 68.324 & 23.913 & 27.118 & 28.776 & 29.819 \\
\hline 768 & 211.635 & 230.232 & 237.844 & 237.046 & 55.530 & 64.898 & 66.406 & 69.535 & 23.919 & 27.029 & 28.304 & 30.396 \\
\hline 896 & 212.862 & 226.003 & 236.943 & 239.088 & 57.570 & 63.319 & 66.248 & 70.740 & 24.190 & 28.046 & 27.625 & 29.441 \\
\hline 1024 & 217.467 & 238.524 & 237.951 & 237.864 & 58.094 & 63.704 & 67.267 & 68.112 & 25.892 & 28.815 & 29.292 & 30.155 \\
\hline 1152 & 219.878 & 236.314 & 241.227 & 241.468 & 59.407 & 65.329 & 66.248 & 67.834 & 26.206 & 28.531 & 29.475 & 29.580 \\
\hline 1280 & 225.054 & 238.249 & 236.436 & 240.509 & 60.368 & 68.467 & 68.262 & 66.184 & 27.283 & 28.576 & 29.198 & 30.096 \\
\hline 1408 & 229.814 & 236.050 & 237.765 & 240.726 & 60.466 & 67.011 & 66.248 & 65.806 & 27.467 & 29.393 & 29.475 & 28.968 \\
\hline 1536 & 230.598 & 238.321 & 237.439 & 239.829 & 62.644 & 67.143 & 68.199 & 66.950 & 27.738 & 30.631 & 29.221 & 29.070 \\
\hline 1664 & 230.325 & 236.821 & 238.680 & 237.799 & 62.350 & 69.362 & 67.063 & 68.749 & 27.560 & 29.998 & 31.291 & 30.661 \\
\hline 1792 & 232.507 & 237.646 & 238.216 & 239.489 & 63.386 & 69.527 & 67.785 & 66.105 & 27.640 & 30.051 & 29.475 & 30.395 \\
\hline 1920 & 234.596 & 236.108 & 239.904 & 239.037 & 63.088 & 66.248 & 66.656 & 66.410 & 28.497 & 30.374 & 30.230 & 31.260 \\
\hline 2048 & 240.823 & 239.421 & 239.292 & 238.537 & 66.690 & 69.230 & 67.343 & 67.113 & 27.643 & 30.783 & 30.119 & 28.811 \\
\hline
\end{tabular}

Table 13: Throughput of a SPAKE2+ (kops/s)

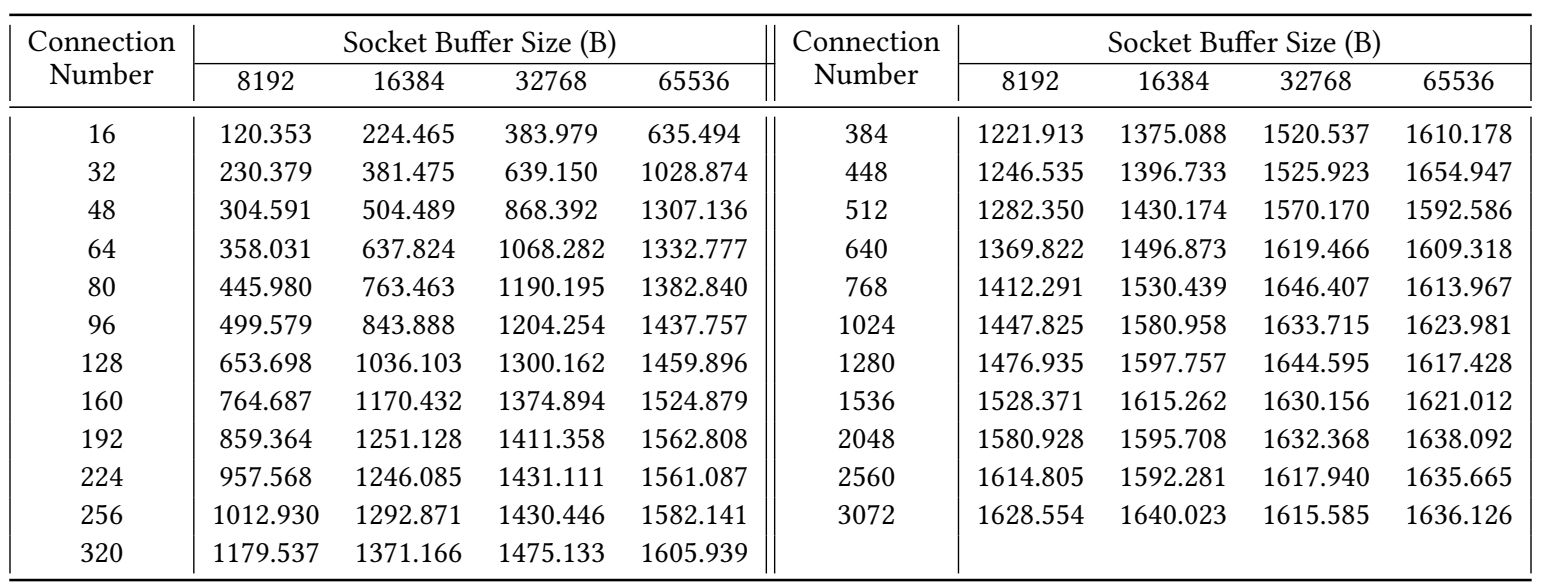


Table 14: Latency of SRP-1024 during Idle/Busy-Hours

\begin{tabular}{|c||c|c|c|c|c|c|c|c|c|c|c|c|}
\hline & $T_{W B 1}(\mathrm{~ms})$ & $T_{W S 1}(\mathrm{~ms})$ & $T_{P S}(\mathrm{~ms})$ & $T_{W S 2}(\mathrm{~ms})$ & $T_{W B 2}(\mathrm{~ms})$ & $T_{W B 3}(\mathrm{~ms})$ & $T_{W S 3}(\mathrm{~ms})$ & $T_{W B 4}(\mathrm{~ms})$ & $T_{\text {total }}^{*}(\mathrm{~ms})$ & $T_{\text {total }}(\mathrm{ms})$ \\
\hline Chrome & $125 / 132$ & $0 / 0$ & $1 / 228$ & $0 / 0$ & $145 / 137$ & $0 / 0$ & $0 / 0$ & $0 / 0$ & $271 / 497$ & $284 / 512$ \\
Firefox & $150 / 154$ & $0 / 0$ & $1 / 191$ & $0 / 0$ & $177 / 187$ & $0 / 0$ & $0 / 0$ & $0 / 0$ & $328 / 532$ & $343 / 548$ \\
IE & $274 / 283$ & $0 / 1$ & $1 / 215$ & $0 / 0$ & $363 / 344$ & $0 / 1$ & $0 / 0$ & $0 / 0$ & $638 / 844$ & $648 / 853$ \\
Microsoft Edge & $109 / 111$ & $0 / 0$ & $1 / 213$ & $0 / 0$ & $133 / 134$ & $0 / 1$ & $0 / 0$ & $0 / 0$ & $243 / 459$ & $252 / 469$ \\
\hline Huawei Browser & $170 / 116$ & $0 / 0$ & $1 / 201$ & $0 / 0$ & $175 / 181$ & $1 / 0$ & $0 / 0$ & $0 / 0$ & $347 / 498$ & $223 / 264$ \\
Firefox for Android & $99 / 100$ & $0 / 0$ & $2 / 176$ & $0 / 0$ & $121 / 142$ & $1 / 0$ & $0 / 0$ & $0 / 0$ & $223 / 264$ & $418 / 472$ \\
\hline
\end{tabular}

"Busy-Hours" means reaching max throughput (240Kop/s) here

$T_{\text {total }}^{*}=T_{T 1}-T_{T 4}$, indicating total time without transmission delay

Table 15: Latency of SRP-1536 during Idle/Busy-Hours

\begin{tabular}{|c||c|c|c|c|c|c|c|c|c|c|}
\hline & $T_{W B 1}(\mathrm{~ms})$ & $T_{W S 1}(\mathrm{~ms})$ & $T_{P S}(\mathrm{~ms})$ & $T_{W S 2}(\mathrm{~ms})$ & $T_{W B 2}(\mathrm{~ms})$ & $T_{W B 3}(\mathrm{~ms})$ & $T_{W S 3}(\mathrm{~ms})$ & $T_{W B 4}(\mathrm{~ms})$ & $T_{\text {total }}^{*}(\mathrm{~ms})$ & $T_{\text {total }}(\mathrm{ms})$ \\
\hline Chrome & $352 / 335$ & $0 / 0$ & $2 / 758$ & $0 / 0$ & $377 / 409$ & $0 / 0$ & $0 / 0$ & $0 / 0$ & $731 / 1502$ & $754 / 1518$ \\
Firefox & $451 / 466$ & $0 / 0$ & $2 / 602$ & $0 / 0$ & $514 / 537$ & $0 / 0$ & $0 / 0$ & $0 / 0$ & $967 / 1605$ & $980 / 1621$ \\
IE & $1208 / 1060$ & $0 / 0$ & $3 / 747$ & $0 / 0$ & $1271 / 1201$ & $0 / 1$ & $0 / 0$ & $0 / 0$ & $2482 / 3009$ & $2494 / 3017$ \\
Microsoft Edge & $351 / 342$ & $0 / 0$ & $3 / 559$ & $0 / 0$ & $407 / 397$ & $0 / 0$ & $0 / 0$ & $0 / 0$ & $761 / 1298$ & $771 / 1309$ \\
\hline Huawei Browser & $362 / 362$ & $0 / 0$ & $3 / 701$ & $0 / 0$ & $408 / 419$ & $0 / 0$ & $0 / 0$ & $1 / 0$ & $774 / 1482$ & $893 / 1592$ \\
Firefox for Android & $310 / 296$ & $0 / 0$ & $2 / 549$ & $0 / 0$ & $346 / 401$ & $0 / 0$ & $0 / 0$ & $0 / 0$ & $658 / 1246$ & $730 / 1338$ \\
\hline
\end{tabular}

"Busy-Hours" means reaching max throughput (70Kop/s) here

$T_{\text {total }}^{*}=T_{T 1}-T_{T 4}$, indicating total time without transmission delay

Table 16: Latency of SRP-2048 during Idle/Busy-Hours

\begin{tabular}{|c||c|c|c|c|c|c|c|c|c|c|}
\hline & $T_{W B 1}(\mathrm{~ms})$ & $T_{W S 1}(\mathrm{~ms})$ & $T_{P S}(\mathrm{~ms})$ & $T_{W S 2}(\mathrm{~ms})$ & $T_{W B 2}(\mathrm{~ms})$ & $T_{W B 3}(\mathrm{~ms})$ & $T_{W S 3}(\mathrm{~ms})$ & $T_{W B 4}(\mathrm{~ms})$ & $T_{\text {total }}^{*}(\mathrm{~ms})$ & $T_{\text {total }}(\mathrm{ms})$ \\
\hline Chrome & $758 / 756$ & $0 / 0$ & $4 / 1552$ & $0 / 0$ & $994 / 914$ & $0 / 2$ & $0 / 0$ & $0 / 0$ & $1756 / 3224$ & $1773 / 3241$ \\
Firefox & $990 / 1016$ & $0 / 0$ & $3 / 1568$ & $1 / 0$ & $1210 / 1137$ & $0 / 1$ & $0 / 0$ & $1 / 0$ & $2205 / 3722$ & $2296 / 3739$ \\
IE & $2742 / 2400$ & $0 / 0$ & $3 / 982$ & $0 / 0$ & $3034 / 2678$ & $0 / 1$ & $0 / 0$ & $0 / 0$ & $5779 / 6061$ & $5790 / 6071$ \\
Microsoft Edge & $781 / 732$ & $0 / 0$ & $3 / 1486$ & $1 / 0$ & $823 / 822$ & $0 / 6$ & $0 / 0$ & $0 / 0$ & $1608 / 3046$ & $1618 / 3055$ \\
\hline Huawei Browser & $798 / 779$ & $0 / 0$ & $4 / 1414$ & $0 / 1$ & $844 / 1004$ & $2 / 1$ & $0 / 0$ & $0 / 0$ & $1648 / 3199$ & $1740 / 3405$ \\
Firefox for Android & $684 / 726$ & $1 / 1$ & $3 / 1343$ & $0 / 0$ & $743 / 830$ & $1 / 1$ & $1 / 0$ & $0 / 1$ & $1433 / 2902$ & $1521 / 3055$ \\
\hline
\end{tabular}

"Busy-Hours" means reaching max throughput (30Kop/s) here

$T_{\text {total }}^{*}=T_{T 1}-T_{T 4}$, indicating total time without transmission delay

Table 17: Latency of SPAKE2+ during Idle/Busy-Hours

\begin{tabular}{|c||c|c|c|c|c|c|c|c|c|c|}
\hline & $T_{W B 1}(\mathrm{~ms})$ & $T_{W S 1}(\mathrm{~ms})$ & $T_{P S}(\mathrm{~ms})$ & $T_{W S 2}(\mathrm{~ms})$ & $T_{W B 2}(\mathrm{~ms})$ & $T_{W B 3}(\mathrm{~ms})$ & $T_{W S 3}(\mathrm{~ms})$ & $T_{W B 4}(\mathrm{~ms})$ & $T_{\text {total }}^{*}(\mathrm{~ms})$ & $T_{\text {total }}(\mathrm{ms})$ \\
\hline Chrome & $48 / 47$ & $0 / 0$ & $1 / 101$ & $0 / 0$ & $56 / 70$ & $10 / 10$ & $5 / 5$ & $0 / 0$ & $120 / 233$ & $134 / 248$ \\
Firefox & $64 / 66$ & $0 / 0$ & $1 / 108$ & $0 / 0$ & $81 / 85$ & $15 / 15$ & $5 / 5$ & $0 / 0$ & $166 / 279$ & $181 / 296$ \\
IE & $199 / 192$ & $0 / 0$ & $1 / 99$ & $0 / 0$ & $230 / 252$ & $63 / 63$ & $5 / 5$ & $0 / 0$ & $498 / 611$ & $510 / 623$ \\
Microsoft Edge & $57 / 46$ & $0 / 0$ & $1 / 100$ & $0 / 0$ & $74 / 68$ & $9 / 10$ & $5 / 5$ & $1 / 0$ & $146 / 229$ & $154 / 240$ \\
\hline Huawei Browser & $51 / 84$ & $0 / 0$ & $1 / 113$ & $0 / 0$ & $76 / 106$ & $11 / 12$ & $5 / 5$ & $1 / 0$ & $145 / 320$ & $191 / 385$ \\
Firefox for Android & $53 / 57$ & $0 / 0$ & $1 / 100$ & $0 / 0$ & $65 / 108$ & $14 / 15$ & $5 / 5$ & $0 / 0$ & $138 / 285$ & $192 / 331$ \\
\hline
\end{tabular}

"Busy-Hours" means reaching max throughput (1600Kop/s) here

$T_{\text {total }}^{*}=T_{T 1}-T_{T 4}$, indicating total time without transmission delay

Table 18: Latency of Hash-based Authentication

\begin{tabular}{|c||cccc|cc|}
\hline & Chrome & Firefox & IE & Microsoft Edge & Huawei Browser & Firefox for Android \\
\hline Delay without $T_{T}(\mathrm{~ms})$ & 61 & 61 & 62 & 61 & 61 & 61 \\
Total Delay (ms) & 73 & 74 & 68 & 70 & 80 & 85 \\
\hline
\end{tabular}

